\title{
Artsibo at Sineng Bayan: Pagpapanatili ng Kolektibong Alaala at Patuloy na Kolektibong Pagsalungat sa Kasinungalingan at Panunupil Rosemarie Roque
}

Bahagi ang Sineng Bayan ng alternatibo at independiyenteng sinema sa ating bansa. Alternatibo ito dahil kumakatawan ito ng mga pelikulang politikal na hindi nakukupot sa komersiyal na interes. Mahalagang usapin ang ganitong paggawa ng mga likhang pampelikula para sa mas seryoso't mas malalim na sinemang Pilipino.

Umusbong ang Sineng Bayan noong dekada 1980 sa panahon ng diktadurang Marcos. Tampok sa politikal na kolektibong pampelikula na umiral sa panahong ito ang AsiaVisions Media Foundation (AVMF), isang organisasyong di-panggobyerno na naglayong gamitin ang pelikula para sa propagandaedukasyon. Kaagapay nito ang kaadbokasiyang Alternative Horizons (AlterHorizons), ang kaunaunahang kooperatibang pangmidya sa bansa.

Ambag ang pag-aaral at kaalaman hinggil sa Sineng Bayan sa pagsulong ng diskurso kaugnay ng pelikula bilang isang mabisang sandatang pangkultura ng mamamayang Pilipino sa kanilang mithiin para sa pambansang kalayaan at tunay na demokrasya.

Pinapahalagahan ang mga likha ng Sineng Bayan bilang pamanang dokumentaryo ng ating bayan. Ang artsibo (archive) ng mga likha ng mga politikal na kolektibong pampelikula ay isang kongkretong bahagi ng kolektibong alaala nating mga mamamayang Pilipino.

Mga susing salita: Artsibong Bayan, Sineng Bayan, Alternatibong Sinema, Politikal na Kolektibong Pampelikula, Pamanang Dokumentaryo

Sineng Bayan (People's Cinema) is a salient part of Philippine alternative cinema, which is in stark contrast to the dominant commercial cinema. It is an important aspect in the pursuit of a more serious Philippine cinema. Political film collectives that arose in the early 1980 s and flourished during the Marcos dictatorship gave way to Sineng Bayan.

This article focuses on the archival audiovisual works of AsiaVisions Media Foundation (AVMF), a non-government organization which primarily utilized film documentaries in its propaganda-education work, and Alternative Horizons (AlterHorizons), the first media cooperative in the country.

This study on Sineng Bayan and the Archives forwards the discourse on film as an effective cultural weapon of the Filipino people in their struggle for national freedom and genuine democracy. Capturing the people's experiences and struggles through audio-visual presentations, videos, and films, the works of these political film collectives are part of the collective memory and documentary heritage of the country.

Key Words: People's Archives, People's Cinema, Alternative Cinema, Political Film Collectives, Documentary Heritage 
Ang konsepto ng "People's Cinema" (o Sineng Bayan) ay unang nagamit sa nakalimbag na literatura ni Roy Iglesias sa kaniyang artikulo noong $1984 \mathrm{sa}$ Sunday Malaya na pinamagatang "An emerging People's Cinema."

Pinili ang Sineng Bayan bilang tumbas ng People's Cinema dahil mas popular, karaniwan, at palasak ang gamit ng salitang "sine" kompara sa "sinema" sa kulturang Filipino. Naging konsiderasyon din, halimbawa, ang mga akda ng mga eksperto sa larangan sa pagpili nila ng salitang "sine" tulad ng librong Sining ng sineng Filipino (2009) ng Young Critics Circle at artikulong "Basahin ang sine" sa Re-viewing Filipino cinema (2011) ni Bienvenido Lumbera sa pagkiling sa tumbasang nabanggit.

Kung pagbabatayan din ang pinagmulan ng "sine," naitala ni Nick Deocampo (2011) sa "Colonial beginnings of native cinema," ang introduksiyon ng kaniyang librong Film: American influences on Philippine cinema na sa pagpasok ng aparatong ginagamit sa pagkuha ng mga imaheng gumagalaw o "motion picture apparatus," pinangalanan ito ng samotsari kasama na ang katawagang "kronofotografo" at, sa kinalaunan, ang "cinematografo," na pinaikli sa "cine" o "sine" nang hiramin para sa wikang bernakular (pp. 14, 26).

Ngunit kung tutuusin, masasabi ring pinagmulan din ng salitang "cinema" o "sinema" ang salitang cinematografo, maaaring maitumbas din ang People's Cinema o Sinemang Bayan (Sineng Bayan).

Higit sa lahat, matutuklasan sa daloy ng pagsasaliksik na ginamit ng ilan sa mga politikal na kolektibong pampelikula (KP) na saklaw ng pagaaral, sa partikular ang grupong nanguna sa larangan na ito, ang AsiaVisions Media Foundation (AsiaVisions o AVMF), ang Sineng Bayan para sa pagpapalaganap at pagpapalabas nito ng mga likhang pelikula/video/ audio-visual presentation (AVP) sa iba't ibang komunidad na naaabot ng grupo. Gayundin, matutuklasang ginamit ito ng kasunod na politikal na KP partikular ng Southern Tagalog Exposure (STeXposure o STeX) para sa parehong aktibidad at layunin (A. Saturay, personal na komunikasyon, 15 Pebrero 2007; K. L. Dalena, personal na komunikayon, 10 Abril 2012; Coronel, 2004).

Ang mga politikal na kolektibong pampelikula na saklaw sa pag-aaral bilang bahagi ng Sineng Bayan ay nakapuwesto bilang parte ng mas malawak na alternatibong sinema sa bansa. Sinasabi na ang alternatibong pelikula ay kilala rin bilang independiyenteng pelikula na sumasaklaw sa mga likhang ginawa nang walang kapital, makinarya, o impluwensiya tulad ng makikita sa mga komersiyal na pelikula. ${ }^{1}$ Hindi nakukupot o hindi nalilimita ng komersiyal na interes sa paggawa ng pelikula at umiiral sa labas ng komersiyal na filmmaking ang alternatibong sinema ${ }^{2}$ (Deocampo, 1994). 
Isang malaking salalayan din ng pag-aaral ang inilatag na katawagan ni Rolando Tolentino kaugnay ng mga politikal na KP (Tolentino, 2008a; Tolentino, 2008b; Tolentino, 2009a; Tolentino, 2009b).

Isinalaysay ni Tolentino (2009a) na ang mga politikal na KP ay integral na bahagi ng indie cinema movement sa bansa at may ugnay sa mga grupong cause-oriented o progresibong kilusan ng mamamayan sa bansa. ${ }^{3}$

Malinaw ang ideolohikal at politikal na pakikipag-ugnayan ng mga KP na sinasaklaw ng pag-aaral sa kilusang masa. Umiiral sila bilang mga kaadbokasiya, kalahok, tagasuporta, at tagatapagtangkilik ng kilusang masa.

Mahahawig ito sa kolektibong pampelikulang produksiyon na tinukoy nina Bordwell at Thompson (1997) sa Film art: An introduction tungkol sa mga kilusang politikal na lumahok sa paggawa ng pelikula. Hinalimbawa rito ang Pransiya noong dekada 1960, nagsulong ng kolektibong pampelikulang produksiyon sa pamamagitan ng Society for the Launching of New Works, isang kooperatiba tungkol sa mga pakikibakang politikal sa pandaigdigang saklaw, tulad ng pagdodokumento ng mga welga at gawaing pang-unyon. Sa Estados Unidos naman, pinakakilala nina Bordwell at Thompson (1997) ang Newsreel group na itinatag noong 1967 upang idokumento ang kilusang protesta ng mga estudyante. Hindi lamang paglalatag ng kolektibong produksiyon ang nagawa ng grupo, kundi maging paglikha ng isang network para sa kolektibong distribusyon ng mga likhang pelikula para sa mga lokal na aktibista. Sa mga nasabing karanasan ng kolektibong produksiyon, may pantay-pantay na paglahok ang maraming manggagawang pampelikula sa paggawa ng proyekto.

Noong panahon ng diktadurang Marcos, ayon kay Deocampo (1994), sumibol ang isang radikal na midya dahil sa matinding tensiyong panlipunan bunga ng diktadura. Nagresulta ito ng paglikha ng mga alternatibong modelo ng pag-iisip at praktika, na umiral sa labas ng mga midya na kontrolado ng estado. Ngunit matapos ang Pag-aalsang Bayan (People Power) noong 1986, marami ang nabigo at nadesmaya nang hindi natupad ang mga inasahang repormang panlipunan pagkabagsak ng diktadurang Marcos. Mula rito, ayon din kay Deocampo, sumulpot ang mga bagong filmmaker na naghanap ng bagong pamamaraan sa pagtakda ng sinema sa bansa. Ayon sa kaniya, inilahad ang tunggalian sa lipunan sa mga short independent film na oposisyonal ang kaibuturan.

Noong dekada 1980, kasabay ng produksiyon ng mga short film sa bansa ang pagtatayo ng mga cause-oriented group at grupong di-panggobyerno (non-governmental organization o NGO) (Deocampo, 1994).

Isa sa tinukoy ni Deocampo (1994) ang Communication Foundation for Asia $(\mathrm{CFA})^{4}$ na gumawa ng mga pelikula sa labas ng industriya at sumubok 
na sumalunga sa mga impormasyong idinidikta ng diktadurang Marcos. Nakapagprodyus ang CFA ng mga media material, kasama rito ang Sugat sa ugat, 1980, ni Ishmael Bernal. Ayon pa kay Deocampo, ilan sa mga pinakadakilang ambag ng CFA ay ang mga makabuluhang dokumentaryo na A spark of courage, 1984; Children of the regime, 1985; at People Power Revolution: The Philippine experience, 1986. Dagdag niya, noong 1985, inilimbag ng CFA ang pioneering book na Short film: Emergence of a new Philippine cinema (Deocampo, 1994).

Isa ring binanggit ang NGO na AsiaVisions na nagprodyus ng mga progresibong dokumentaryo (Deocampo, 1994). Itinatag noong 1982, idinokumento ng AsiaVisions ang pakikibaka para sa katarungan at demokrasya noong mga huling taon ng diktadurang Marcos.

Ilan sa mga makabuluhang likha ng grupo, sa pagtatala ni Deocampo (1994), ang Arrogance of power, 1983; Lakbayan, 1984; at No time for crying, 1986.

Kaugnay sa mga isinaad ni Deocampo, ayon kay Nicanor Tiongson (2001), sumibol noong dekada 1980 ang isang mayabong at mapagpasiyang kilusan sa paggawa ng pelikula.

Matapos ang asasinasyon kay Sen. Benigno Aquino noong 1983, may ilan sa mga independiyenteng filmmaker na mas nagdadala ng kamulatang panlipunan ang lumikha ng Sineng Bayan para ilantad ang mga katotohanang sinikil ng midya at organisasyong maka-Marcos (sipi kay Iglesias ni N.G. Tiongson, 2001). Karamihan sa mga independiyenteng filmmaker na nabanggit ni N.G. Tiongson ay bahagi ng AsiaVisions.

Ang pagsibol na ito ng People's Cinema, ayon kay Roy Iglesias (1984, p.7) sa kaniyang artikulo sa Sunday Malaya na pinamagatang "An emerging People's Cinema," ay bunga ng lumalakas na kilusang protesta noong panahong nabanggit na humubog sa filmmaking bilang isang "guerilla art" sa kamay ng mga progresibong artista. Ang katotohanan na nairerekord ng pelikula at video ay naging "pinakaepektibong propaganda" (Iglesias, 1984, p.7). Ang patuloy na paglaganap ng makamamamayang pelikula at video, dagdag ni Iglesias, ay nagpapahiwatig na nagkaroon na ng pambansang kahalagahan ang pakikibaka para sa kalayaan at demokrasya para mahikayat ang mga film artist sa imahen ng kontemporaneong realidad. Ito ang tanda ng simula ng isang Sineng Bayan na may kolektibong tagapagtangkilik (Iglesias, 1984).

Napaunlad ang konsepto ng People's Cinema sa konteksto noong panahon ng diktadurang Marcos, bilang alternatibo sa telebisyon at pelikula. Sa panayam kay Iglesias (personal na komunikasyon, 16 Enero 2012), sinabi niya na dahil sa kontrolado ng estado ang midya, ang tanging paraan para bigyang-pansin ng midya ang nangyayari sa paligid ay kung 
matitiyak na "naisasama ang katotohanan" sa mga naibobrodkast, tulad ng maganap ang asasinasyon kay Sen. Benigno Aquino. Ayon sa kaniya, walang nagawa ang midya noon kundi ipamalita ang pangyayari dahil napakalaki ng penomenong iyon. Sa sobrang laki ng isyu, hindi na maaaring balewalain ito ng midyang kontrolado ng estado. Kaya't kung ang mamamayan mismo ang gagawa ng realidad, ang mamamayan mismo ang lalahok sa pagprotesta, walang magagawa ang midya kundi ibalita ang katotohanan na ito. Sa pananaw ni Iglesias, usapin ang pagtustos ng mamamayan sa sarili nilang sinema. Nakita sa panahon ng diktadura na nagawa ito sa pamamagitan ng dokumentasyon ng mga karanasan ng mamamayan gamit ang video. Malaking bagay na mas mura ang video kaysa pelikula. Kaya't nagkaroon ng kapangyarihang sinematiko ang ordinaryong mamamayan dahil sa video (R. C. Iglesias, personal na komunikasyon, 16 Enero 2012).

Sinasabi mang kolektibong hakbangin ang paggawa ng pelikula, naiiba pa rin ang paglikha ng pelikulang mainstream sa pelikulang saklaw ng People's Cinema dahil may malinaw na kolektibong kamulatan na daladala ang mga pelikulang nakapaloob sa People's Cinema. Ayon kay Iglesias (personal na komunikasyon, 16 Enero 2012), silang mga filmmaker ay mulát na bahagi ng isang kolektibo sa isang kilusan. Mulát sila na sila'y bahagi ng isang sinemang may tiyak na layunin o tinaguri niya itong purposive cinema. Tangan nila ang isang ideolohiyang bayan ("people's ideology"), isang uri ng ideolohiyang mapagpalaya ("liberating type of ideology"). Isa itong alternatibo sa midya ng estado lalo't sa panahon na walang pagkukunan ng balita, istorya, o kuwento ang taumbayan kundi ang midyang kontrolado ng estado.

Higit sa lahat, malinaw na tinutukoy ng pananaliksik na ito ang Sineng Bayan bilang mga pelikula, video, at iba pang AVP na makamamamayan at naglalaman ng mga kontemporaneong realidad sa gitna ng pakikibaka ng mamamayan para sa kalayaan at demokrasya.

Nagsisilbi ang mga likha ng mga politikal na kolektibong pampelikula bilang imbakan ng mga alaala ng ating mamamayang buong giting at tapang na sumasalungat sa kasinungalingan at panunupil na namamayani sa lipunan. Kaya't gumagampan ng panlipunang kabuluhan ang artsibong awdyo-biswal bilang imbakan at balon ng kongkretong batayan ng kasaysayan ng bayan. Isa na rito ang artsibong awdyo-biswal ng AsiaVisions Media Foundation bilang pangunahing politikal na kolektibong pampelikula ng dekada 1980 na nagdokumento ng mga karanasan ng kilusan at pakikibakang masa ng nasabing panahon lalo na sa panahon bago at pagkatapos bumagsak ang diktadurang Marcos. 


\section{Sineng Bayan at tradisyong pampelikula ng AsiaVisions at AlterHorizons}

Mahalaga ang koleksiyon ng mga nagawang pelikula ng AsiaVisions sa loob ng dekada 1980, ang partikular na dekada kung kailan ito naitatag at nagpalakas bilang isang organisasyong pampelikula na may adbokasiyang politikal.

Ang AsiaVisions ang tinukoy ni Rolando Tolentino (2009a) na orihinal na politikal na kolektibong pampelikula na integral na bahagi ng indie cinema movement sa bansa at may ugnay sa mga grupong cause-oriented o progresibong kilusan ng mamamayan sa bansa.

Kaugnay nito, ayon kay Bonifacio Ilagan sa First National Conference on Alternative Media noong 10 Oktubre 2014 sa kaniyang panayam na "Progressive video groups: History, lessons, and challenges," may dalawang pioneer na video group noong dekada 1980 ang natatag na pangunahing nilayon na "gamitin ang video bilang kasangkapan ng kilusang masa sa noo'y umaarangkadang laban ng taumbayan kontra sa rehimeng Marcos" (Ilagan, 2014, p.1). AsiaVisions at Alternative Horizons (AlterHorizons) ang dalawang grupong tinutukoy.

Itinatag ang AsiaVisions nina Lito Tiongson, Jose "Jocua" Cuaresma, at Danny Consumido nang huling bahagi ng $1982,{ }^{5}$ na malinaw na gumamit ng dokumentaryo sa kalakhan sa pagsalunga sa organisado at laganap na propaganda ng noo'y gobyernong Marcos. Una itong nakilala bilang Creative Audio Visual Specialists (CAVS) (N.G. Tiongson, 1984).

Si Lito Tiongson ang tumayong kauna-unahang executive director ng AsiaVisions (AsiaVisions Media Foundation, 1985). Siya rin ang tumayong board secretary partikular nang nagparehistro ang grupo sa Securities and Exchange Commission(SEC) noong 1985. Kasama niL. Tiongson sa nailistang mga miyembro ng Board of Directors ng AsiaVisions sa rehistrasyon sina Lino Brocka, Jose Lacaba, Romeo Royandoyan, Lutgardo Labad, at Danilo Consumido (AsiaVisions Media Foundation, 1985). ${ }^{6}$ Kinalaunan, magiging bahagi rin ng Board sina Renato "RC" Constantino, Bienvenido Lumbera, at Jose "Pete" Lacaba (A. Saturay, personal na komunikasyon, 15 Pebrero 2007).

Samantala ang AlterHorizons, na pinangunahan nina Jose Cuaresma at Bobby Roldan, ay sinimulang inorganisa bilang isang kooperatiba noong 1986 (J. Cuaresma, personal na komunikasyon, 20 Marso 2012). Ito ang maituturing na kauna-unahang media cooperative sa bansa.

Isa sa mga unang nagsilbing miyembro ng Board of Directors ng AlterHorizons sina Ricky Lee at Pio de Castro, kasama nina Jose Cuaresma at Bobby Roldan (J. Cuaresma, personal na komunikasyon, 20 Marso 2012). 
Sa mga nasabing batayan tumutungtong ang sanaysay sa pagbibigay ng tuon sa dalawang organisasyon. Sa pangkalahatan, ang layunin sa pag-aaral sa mga politikal na kolektibong pampelikula ay upang patingkarin ang usapin ng paglikha ng mga kolektibong pampelikula ng mga pelikula, video, o AVP bilang isang organisasyon taliwas sa dominanteng kalakaran sa komersiyal at mainstream na pelikulang sumesentro lamang sa indibidwal na direktor (indibidwal bilang auteur).

Sa Short film: Emergence of a new Philippine cinema (Deocampo, 1985) at "Alternative cinema" (Deocampo, 1994) sa Volume VIII ng CCP Encyclopedia of Philippine Art bilang ilang halimbawa, mapapansin na nakasentro ang mga ito sa mga indibidwal na filmmaker. Dahil dito, mas nagbibigay ng pangunahing pagpapahalaga ang kasalukuyang pag-aaral na ito sa mga grupo o kolektibong lumilikha ng pelikula, video, at AVP_mga grupo sa video at pelikula na "hindi puwedeng hiwalay sa malawak na kolektibo ng kilusang masa" na "hindi maaaring... magsarili, gaya ng mga burges na artista sa kanilang walang-hanggang paghahanap ng inspirasyon at paghahabol sa The Great Filipino Film" (Ilagan, 2014, p.3). Nasa diwa ito ng pagpapatampok ng papel ng kolektibong pagkilos sa pagyabong ng Sineng Bayan. Gayumpaman, hindi nangangahulugang isinasantabi na ang mga indibidwal na papel at ambag sa pagsulong ng Sineng Bayan.

Tunay na mayabong ang Sineng Bayan at kabahagi rito ang napakaraming bilang ng mga filmmaker, nakapaloob/pumaloob man sa isang kolektibong pampelikula na bahagi ng isang mas malawak na kilusan masa o nag-(a) ambag bilang indibidwal para sa causa ng isang mas malawak na kilusang masa. Bilang delimitasyon sa sanaysay na ito, tiyak na sasaklawin lamang ang mga karanasan at piling pelikulang politikal na likha ng mga kolektibong pampelikula na AsiaVisions at AlterHorizons. Ang mga nababanggit na KP ay kumikilos bilang kolektibo sa konseptuwalisasyon at eksekyusyon ng konsepto at produksiyon ng aktuwal na pelikulang kanilang nilikha hanggang sa eksibisyon at distribusyon ng mga ito. Tuon sa sanaysay na ito ang AsiaVisions, kasama ang AlterHorizons, na may mahigpit at masiglang ugnayan $^{7}$ sa AsiaVisions para ipakita ang tradisyon at politikang nahawan sa diwa ng Sineng Bayan. Sa pamamagitan ng mga kanilang piling likha, partikular ang mga nagawa noong dekada 1980, ipapakita kung paano nakapaghawan ang tradisyon at napanghawakan ang politika ng dalawang KP. Ang kanilang tradisyon at politika'y naipagpapatuloy ng mga sumunod at/o kasalukuyang politikal na kolektibong pampelikula.

Sa panahon ng pagkakatatag at pagsibol, kapuwa tumugon ang AsiaVisions kasama ng AlterHorizons sa mga maiinit ng isyu ng lipunan, dialintana ang posibleng pandarahas at panunupil na maari ding kaharapin sa paglalantad ng tunay na kalagayan ng mamamayan. 
Isang NGO man ang AsiaVisions na may relatibong limitadong bilang ng staff na palagiang direktang kalahok sa buhay at pagpapatakbo ng organisasyon o isang kooperatiba man ang AlterHorizons na may General Assembly na inaasahang mapapalahok sa mga proyekto (M. Francia, personal na komunikasyon, 1 Mayo 2016; M. Bello, personal na komunikasyon, 1 Mayo 2016), ${ }^{8}$ malinaw ang tulungan ng dalawa bilang mga magkaadbokasiya sa layuning gamitin ang midyum ng pelikula at video bilang mga epektibong kasangkapan para isulong ang interes ng mga mamamayan sa kanilang paglikha ng kanilang kasaysayan.

Sa karanasan ng dalawang kolektibong pampelikula ${ }^{9}$ makikita ang mga nuance sa pag-iral ng AsiaVisions na nagsimula bilang isang "loose formation" (bilang CAVS) noong 1982 hanggang pagiging isang institusyong umiral bilang NGO mula 1985 hanggang huling bahagi ng dekada 1990 at pag-iral ng AlterHorizons mula inisyatibang naisakonsepto bago 1986 patungo sa pagtatayo nito noong 1986 hanggang gitna ng unang dekada ng bagong milenyo, nakapag-ambag ang dalawang kolektibong pampelikula sa pangkalahatang pagsulong ng kilusang masa sa pamamagitan ng mga nalikhang pelikulang politikal na integral na bahagi ng alternatibong sinema sa bansa. Hindi man lahat ng likha ng dalawang grupo ang maituturing na direktang anyo ng Sineng Bayan, o mga pelikulang lantarang nagsusulong at umuugnay sa mga grupong cause-oriented o progresibong kilusan ng mamamayan sa bansa, may puwang at may espasyo ang kasaysayan para kilalanin ang kanilang mga pagsisikap.

Makikita sa mga karanasan ng AsiaVisions, maging ng AlterHorizons, ang mga katangian ng kolektibong produksiyon ${ }^{10}$ sa usapin ng pagpapahalaga at pagbibigay ng pantay na turing sa bawat gawaing pamproduksiyon bilang mahalagang gawain. Makikitang may mga malinaw na hatian man ng gawain sa produksiyon (dibisyon ng gawain), nagsisimula ang lahat sa kolektibo at nagkakaisa nilang pananaw at paninidigan kung para kanino ang kanilang mga likha.

Pinagdedesisyunan nang kolektibo kung ano ang gagawan ng proyekto. Kolektibo silang nagpapasiya sa mahahalagang bahagi ng produksiyon, tulad ng paggawa ng iskrip, at pagharap sa komite at maging sa target audience ng nabuong pelikula o video. Hindi nalilimita ang bawat kasapi, partikular sa karanasan ng AsiaVisions, sa iisang gawain lamang. Hindi sila nagsimula bilang mga filmmaker, nasanay lamang sa proseso ng gawain, at patuloy na umuunlad sa pagganap ng iba pang gawain dahil tinitiyak ang rotasyon sa pagganap sa mga ito.

Sa simula, umasa ang dalawang KP sa sari-sariling gamit at personal na rekurso sa paggawa ng produksiyon hanggang sa nakayang magpundar ng sariling gamit at magkaroon ng mga pagkukunan ng pondo. Sa kaso ng 
AsiaVisions, malaking tulong ang mga grant na natanggap sa pagpupundar na ito, kasama ang kagamitan nila sa Mobile Cinema para sa eksibisyon at pag-abot sa mga manonood sa antas-grassroot.

Hindi man nagsagawa ng audience outreach program ang AlterHorizons, nakatulong nang malaki ang mga narrowcasting ng kanilang mga likha. Gerilyang masasabi sa pag-abot ng lahat ng posibleng bilang ng manonood. Sa konteksto ng kanilang panahon na nakaasa sa magnetic tape ang mga gawa at sa popularidad ng mga VHS o Betamax sa mga kabahayaan lalo sa mga pamilya ng mga OFW, malinaw na pagmaksimisa sa ano ang mayroon ang mahalaga sa pag-abot ng audience. Lalo na ang mga kopya ng Betamax at VHS na mga gawa kapuwa ng AsiaVisions at AlterHorizons na napunta sa mga eskuwelahan, mga unibersidad, at mga aklatan sa iba't ibang bahagi ng bansa ay mahalagang bagay na matuturing.

Mapangahas ang dalawang grupo, lalo na't umiral ang mga ito sa panahon ng diktadurang Marcos na kahit ang simpleng paggawa at panonood ng mga likhang naglalaman ng realidad ng lipunan ay mapapangahas at mapanganib na gawain. Masasabing "maliit man" ang mga produksiyon, hindi tulad ng mga gawa ng mainstream at komersiyal, pero hindi naging maliit ang natugunang interes ng mga likha nila. Hirap man sa pagpipinansiya, distribusyon, at eksibisyon, hindi tulad ng mainstream, hindi naman matatawaran ang ambag ng mga kolektibong produksiyon ng AsiaVisions at AlterHorizons sa pagsulong ng mas malawak na interes ng mamamayan.

Sapaglatagngganitonghistorikal at politikal nakabuluhan maipupuwesto ang malaking papel ng preserbasyon ng at akses sa mga likha ng mga politikal na KP bilang mga pamanang dokumentaryo o documentary heritage ng mamamayang Filipino sa pagtitiyak ng pagpapatuloy ng tradisyon, praktika, at silbi ng Sineng Bayan sa buhay, kultura, pakikibaka, at lipunang Filipino lalo sa panahon ng papaigting na rebisyonismong pangkasaysayan.

Nilalaman ng artikulong ito ang kalakaran at politika ng Sineng Bayan bilang [kontra]diskurso na malinaw na nakasalig at kumikiling sa puntode-bista at paninindigan ng mamamayang nasa ibaba't laylayan ng lipunan. Nilalaman nito ang Sineng Bayan bilang produkto ng kolektibong pagkilos at pagsalungat sa mga kabalintunaan ng naghaharing sistema at hamon ng pagpapanatili ng mga likhang-sining ng mga politikal na kolektibong pampelikula bahagi ng Sineng Bayan bilang mahalagang bahagi ng kolektibong alaala ng mamamayan.

Kasama rito ang reiterasyon na ang kasaysayan at pag-iral ng Sineng Bayan ay malinaw na nakapuwesto sa gitna ng pakikibaka ng mamamayan para sa kalayaan at demokrasya. Bibigyan ng kongklusyon ang kasaysayan ng Sineng Bayan kaugnay hindi lamang ng pangangailangang tumugon at umangkop ng mga politikal na KP sa patuloy na pag-unlad ng teknolohiya 
ng pelikula kundi sa usapin pa ng kung paano nagsisilbing inspirasyon at hamon ang hitik sa karanasan at mapanlabang kasaysayan ng Sineng Bayan sa patuloy na pagsisikhay ng mga politikal na KP na maging aktibong bahagi ng pagsulong ng pakikibaka ng mamamayan para sa kalayaan at demokrasya.

Hindi lamang nagtatala ng nagbabagong kasaysayan ng mamamayan ang mga politikal na kolektibong pampelikula. Bahagi ang mga karanasan at mga likha nito sa nagbabagong kasaysayan.

\section{Sineng Bayan at lkatlong Sinema: Organisasyong pampelikula para sa adbokasiyang politikal}

Sa mga likha ng AsiaVisions maaarok ang esensiya ng Sineng Bayan sa lapit at perspektiba ng Ikatlong Sinema ("Third Cinema"). Isinasaad nina Fernando Solanas at Octavio Gettino (1976) na mga katangian ng Ikatlong Sinema ang pagsasadokumento at paglalantad ng katotohanan at paghahanap ng orihinal na lengguwahe sa paggawa ng pelikula. Katangian din ng Ikatlong Sinema ang paghimok nito sa mga manonood na maging mapagpasiya, kumilos, at maging tagapaglikha ng ideolohiya. Mailalapat ang mga nasabing katangian ng Ikatlong Sinema sa mga katangian ng Sineng Bayan sa ating bansa.

Mahalaga ang koleksiyon ng nagawang pelikula ng AsiaVisions sa loob ng dekada 1980, panahong naitatag at nagpalakas ang AsiaVisions bilang isang organisasyong pampelikula na may adbokasiyang politikal.

Tampok sa mga likha ng AsiaVisions sa loob ng dekada 1980 na bibigyang detalye sa pag-aaral na ito ang ilang video o pelikula na may available na kopyang nasa o nailipat na sa digital format at nagawa ng grupo lalo na noong na dekada 1980 tulad ng Wings of deceit (Creative Audio-Visual Specialists [CAVS], 1982, 20 minuto, orihinal sa Betamax) at Arrogance of power (AsiaVisions, 1983, 38 minuto, orihinal na nasa 8mm) sa panahon $\mathrm{ng}$ diktadurang Marcos; kasama ang No time for crying (AsiaVisions, Christian Aid, \& Tiongson, 1986, 30 minuto, orihinal sa 16mm) at Behind the walls of prison (AsiaVisions, Samahan ng mga Ex-Detainees Laban sa Detensiyon at Aresto [SELDA], \& Tiongson, 1987, 60 minuto, orihinal sa U-matic) na nagawa sa panahon na kababagsak pa lang naman ng rehimeng Marcos. Tampok naman sa likha ng AlterHorizons ang dokumentaryong Edjop (Edgar Jopson Foundation \& Alternative Horizons [AlterHorizons], 1986, 54 minutos, orihinal sa U-Matic).

Mahalagang idiin sa yugtong ito ang malaking papel ng IBON Foundation dahil nasa pangangalaga at receivership nito ang lahat ng natitirang artsibong awdyo-biswal ng AsiaVisions. ${ }^{11}$

Sa isang banda, hindi maitatangging bahagi lamang ang artsibong awdyo-biswal na ito ng pagsasadokumento ng isang hitik at masalimuot 
na yugto ng kasaysayan ng bansa. Gayumpaman, mahalaga at susing salalayan ang koleksiyong awdyo-biswal ng AsiaVisions bilang kongkreto at mababalikang alala natin bilang mga mamamayan. Dahil hindi maitatangging naidokumento ng grupo ang ilang mahahalagang isyu at pangyayaring nagbago sa daloy ng ating kasaysayan, partikular ng dekada 1980, na nilalaman ng mga nagawang pelikula/video ng naturang grupo. Sa ilang likha sa ilalim ng CAVS (naunang pangalan ng AsiaVisions), naging laman ang usapin ng mga base militar ng Amerika sa bansa, maging ang kalagayan at problemang agraryo sa bansa ("Curriculum vitae of Lito Tiongson," n.d.; IBON Video Catalog, 2003). Sa mga sumunod na likha bilang AsiaVisions, laman ng mga likha ng grupo ang mga samot-saring kalagayan at isyu ng panahon. Ilan sa mga ito ay ang mga sumusunod: militarisasyon at paglabag sa mga karapatang pantao sa panahon ng Batas Militar, maging ang paglaban ng mamamayan; problemang agraryo ng mga magsasaka lalo sa Mindanao at pagtatayo nila ng kooperatiba; karanasan ng mga detenidong politikal at kanilang di-pagsuko sa gitna ng kagipitan at pagmamalupit sa kanila ng gobyerno; karanasan ng mga migranteng Filipino at mga gawain ng Bethune House sa Hongkong; taunang protesta na nauwi sa pagmasaker ng mga magsasaka sa Mendiola noong 1987; at pagpaslang sa mga lidermasa tulad nina Lean Alejandro at Ka Lando Olalia (batay sa imbentaryo sa buong koleksiyon na isinagawa mismo ng mananaliksik-manunulat noong Abril hanggang Mayo 2012).

Sa mga piling likhang video at pelikula na saklaw ng artikulong ito, makikita na tumutupad ang AsiaVisions at AlterHorizons sa layuning maisakodokumento at mailantad ang mga katotohanan sa lipunang Filipino, partikular sa panahon ng rehimeng Marcos at Aquino.

\section{Piling likha ng AsiaVisions at AlterHorizons}

Ilan sa mahahalagang nalikha ng AsiaVisions at AlterHorizons partikular noong dekada 1980 ang mga sumusunod:

\section{Wings of Deceit (1982, 20 minuto, orihinal sa Betamax)}

Inilantad ng Wings of Deceit (CAVS, 1982) ang katotohanan hinggil sa 20 base militar ng Amerika sa bansa (na nanatili sa bansa hanggang Setyembre 1991 bago ang abrogasyon ng US-RP Military Bases Agreement, na umiiral sa panahon na ginawa ang video; nais palawigin ang base militar hanggang Oktubre 2018 sa ekstensiyon ng foreign sales credits ng Estados Unidos na inaprobahan ng diktadurang Marcos, ayon sa video.

Tampok din sa video ang Clark Air Field, ang pinakamalaking base militar ng Amerika sa Asya (at mas malaki pa sa District of Columbia ng Estados Unidos), Subic Naval Base, at maging ang ilan pang base militar tulad ng Wallace Station sa Poro Point, Camp John Hay, San Miguel Naval Base, at Bamban Beacon Annex. 


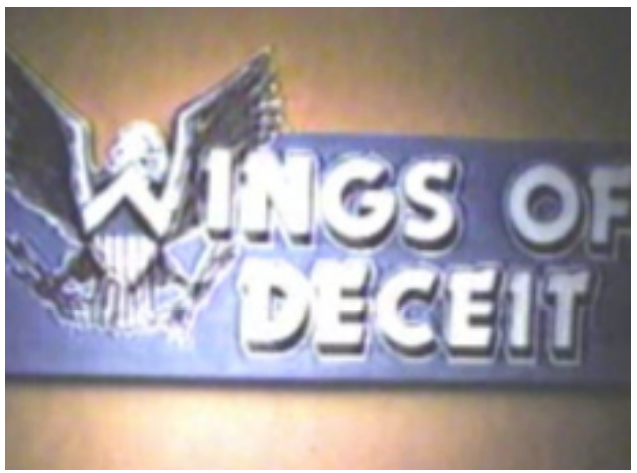

Larawan 1.Snapshot mula sa Wings of Deceit (CAVS, 1982), dokumentaryong mala-slideshow ng CAVS, ang unang pangalan ng AsiaVisions.
Epektibo ang video dahil sa mahusay na pagtitilad ng usapin hinggil sa base militar sa pagbigay ng mga kongkretong datos at pangkasaysayang balangkas ang usapin ng base militar sa bansa. Malinaw ring ipinakita ng video ang papel ng mga base militar sa panunupil na ginawa ni Marcos sa panahon ng Batas Militar at mas maaga pa, noong dekada 1930 sa pagsupil sa mga pag-aalsa ng magsasaka, noong dekada 1950 laban sa mga Huk (Hukbong Bayan

Laban sa Hapon o HUKBALAHAP) hanggang matapos ang deklarasyon ng Batas Militar. Sa pangkalahatan, ipinakita sa video ang papel ng mga US aid at assistance sa nasabing panunupil.

Inilantad din ng video ang kaayusang panlipunan sa bansa na nagpapahintulot, at labag sa soberenya ng bansa, ang pananatili ng bansa militar. Sa partikular tinukoy nito ang kapangyarihan pang-ekonomiya ng U.S. sa bansa at ang papel ng iilang naghaharing uri sa bansa sa pananatili ng dominasyon ng US sa bansa. Ginawa ito ng video sa pamamagitan ng pagbibigay ng mga kongkretong datos sa mga puntong inihahayag nito, maging ang mga kongkretong pagpapalaman sa mga batayan kung bakit dapat tutulan ang pananatili ng base militar sa bansa.

\section{Arrogance of power (1983, 38 minuto, orihinal na nasa $8 \mathrm{~mm})$}

Sa Arrogance of Power (AsiaVisions, 1983), inilantad ang kabalintunaan hinggil sa deklarasyon ni Marcos sa kawalan ng detenidong politikal dahil sa Batas Militar pamamagitan ng paglalantad ng mga paglabag sa karapatang
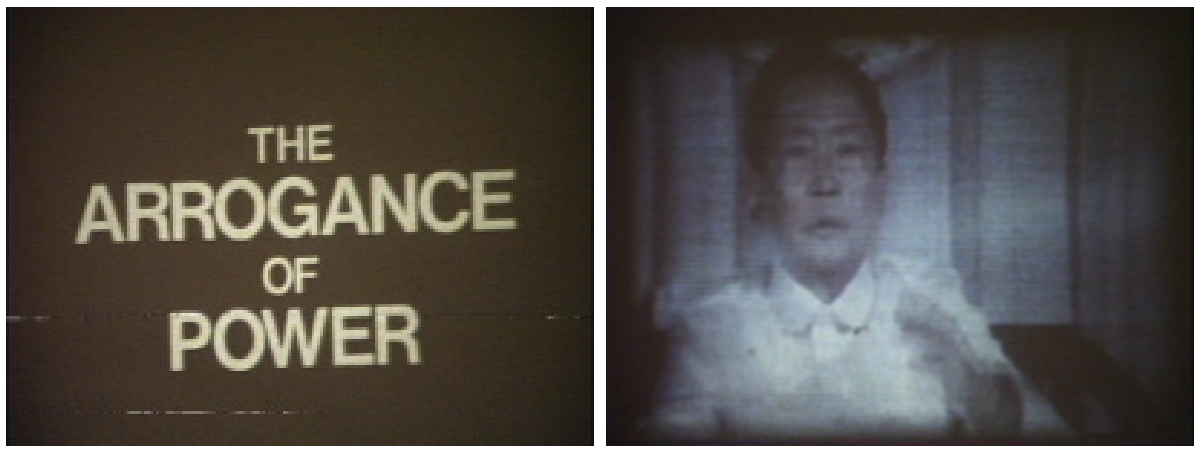

Larawan 2-3. Mga snapshot mula sa Arrogance of Power (1983): Mga imahen mula sa unang isang minuto ng dokumentaryo (kuha sa Super $8 \mathrm{~mm}$ ). 
pantao. Itinampok ng pelikulang ito ang mga kaso nina Manuel Roque, Alim Datulan, Modesto Sison, Fernando Fernandez, at Macli-ing Dulag na pawang mga biktima ng pag-aresto, pagdukot, at pagpatay na pawang itinanggi lahat ni Marcos sa pagsabi na isa lamang ang nahuli sa ilalim ng Batas Militar dahil sa heroin. Itinampok din ng pelikula ang mga ulat ng Amnesty International (AI) at Task Force Detainees of the Philippines (TFDP) hinggil sa mga paglabag sa karapatang pantao na naganap sa ilalim ng at matapos ang Batas Militar (halimbawa ang pagbanggit sa dokumentaryo ng isang ulat ng AI noong 1975). Ipinakita rin ang mga taong simbahan bilang ilan sa mga nabiktima sa ilalim ng batas militar. Pinatampok din ang "hamletting" (kuha ng aktuwal na mga lugar na inilagay sa hamlet), "salvaging," at tortyur na pinakamalaganap sa ilalim ng diktadurang Marcos.

Inihayag din ng pelikula ang malinaw na militarisasyon matapos ang Batas Militar sa pagpapakita ng mga heneral na binigyan ng posisyon sa gobyernong sibil, maging ng papalaking bilang ng militar, pulis, citizens' army, armadong barangay brigade, at iba't ibang ahensiyang paniktik. Ipinakita rin ang ibinigay na kapangyarihang pang-ekonomiya ni Marcos sa mga militar sa pagpasok ng mga ito sa sektor ng pagnenegosyo/bisnes.

Inilantad din ang mga pangako ni Marcos na iimbestigahan ang ulat ng Amnesty International at didisiplinahin ang mga nagkasala sa militar. Pero ayon sa pelikula, naganap man ang imbestigasyon ngunit wala namang findings na isinapubliko at wala ring naparusahang maysala.

Sa pelikula, inilahad na nagkaroon ng imunidad ang mga sundalo laban sa mga kasong isasampa sa kanila kaugnay ng paglabag sa karapatang pantao.

Sa paggamit nito ng aktuwal na interview ng isang Assistant Secretary of State, nalantad ang interes ng Amerika sa Pilipinas at pagsuhay nito sa diktadurang Marcos. Sa nabanggit na panayam sa Assistant Secretary of State, sinabi niya na ang Pilipinas bilang isang dating kolonya ng Amerika ay kumakanlong sa dalawang malaking overseas military base ng Amerika na may estratehikong puwesto sa Silangang Asya at Kanlurang Pasipiko hanggang Dagat Indian at Gitnang Silangan (Kanlurang Asya). Isinaad din sa pelikula na ang mga sundalong Filipino ay replika ng mga naghasa sa kanilang mga sundalong Amerikano sa kontra-insurhensiya. Inilantad din sa pelikula ang direktang pagtulong ng Amerika sa diktadurang Marcos bilang malinaw na pagpapahintulot nito sa pag-iral ng diktadura.

Binigyan din ng konteksto sa kasaysayan ang panunupil na naganap sa ilalim ng diktadurang Marcos sa panunupil na isinagawa ng mga mananakop sa ating bansa, mga metodong mapanupil na ginamit ng mga mananakop na Kastila, Amerikano, at Hapon. 


\section{No time for crying (1986, 30 minuto, orihinal sa $16 \mathrm{~mm}$ )}

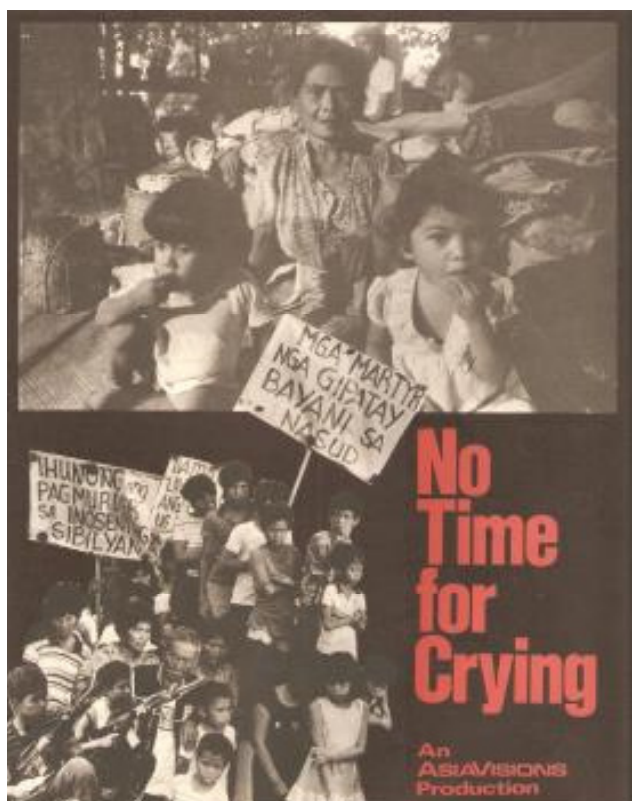

Larawan 4. Kopya ng one sheet ng No Time for Crying (AsiaVisions, Christian Aid, \&Tiongson, 1986).
Isinadokumento ng AsiaVisions sa pamamagitan ng No Time for Crying (AsiaVisions, Christian Aid, \& Tiongson, 1986) ang kalagayan ng mga mamamayan sa Agusan del Norte, partikular sa Lungsod Butuan at sa Munisipyo ng Magallanes. Inilantad dito ang paglabag sa karapatang pantao naganap noong diktadurang Marcos at pumalit na gobyernong Aquino. Nagsimula ang pelikula sa lamay ng pinatay na lider ng maralitang tagalungsod at unyonista (Toto Senador) tatlong buwan bago bumagsak ang rehimeng Marcos.

Ipinakita rin sa No Time for Crying ang papel ng mga taong tagasimbahan sa pagtulong sa pagtatayo ng mga kooperatiba ng mga mahihirap na mamamayan ng Lungsod Butuan (Christian Aid para sa negosyong rice cake ng kababaihan sa Butuan) at Magallanes (United Church of Christ in the Philippines para sa paggawa ng uling para sa mga nawalan ng trabaho). Naipatampok dito ang naganap na "lock-up" ng isang kompanya sa pagtotroso na nagresulta ng biglaang kawalan ng hanapbuhay ng 15,000 pamilya at nag-iwan ng mayroong 40 milyong pisong utang sa mga manggagawa sa porma ng hindi binayarang back wages. Itinampok din dito ang pang-aagaw ng lupa ng isang korporasyong multinasyonal na nangakong bibigyan ng kompensasyon ang mga magsasakang nawalan ng lupa at ginawa nitong mangagagawa ngunit hindi nagbayad at kinalauna'y hindi na tumutupad sa batas sa paggawa, na naganap sa ibang bahagi ng Agusan del Norte. Narito rin ang pagtulong ng taong tagasimbahan para sa pagtatayo ng "rice bank" para makatulong sa komunidad.

Higit sa lahat, ipinakita sa No Time for Crying ang tapang ng mga naabusong mamamayan na ireklamo ang nang-abusong pulis na ilegal na nang-aresto sa isang magsasakang pinaratangang suporter ng NPA. Naidokumento ng pelikula ang paghaharap ng mamamayan at ng meyor ng Magallanes kaugnay ng reklamo. Naipakita ng kanilang sama-samang pagtugon sa usapin ay nagbunga ng taktikal na katugunan sa kanilang hinaing. 

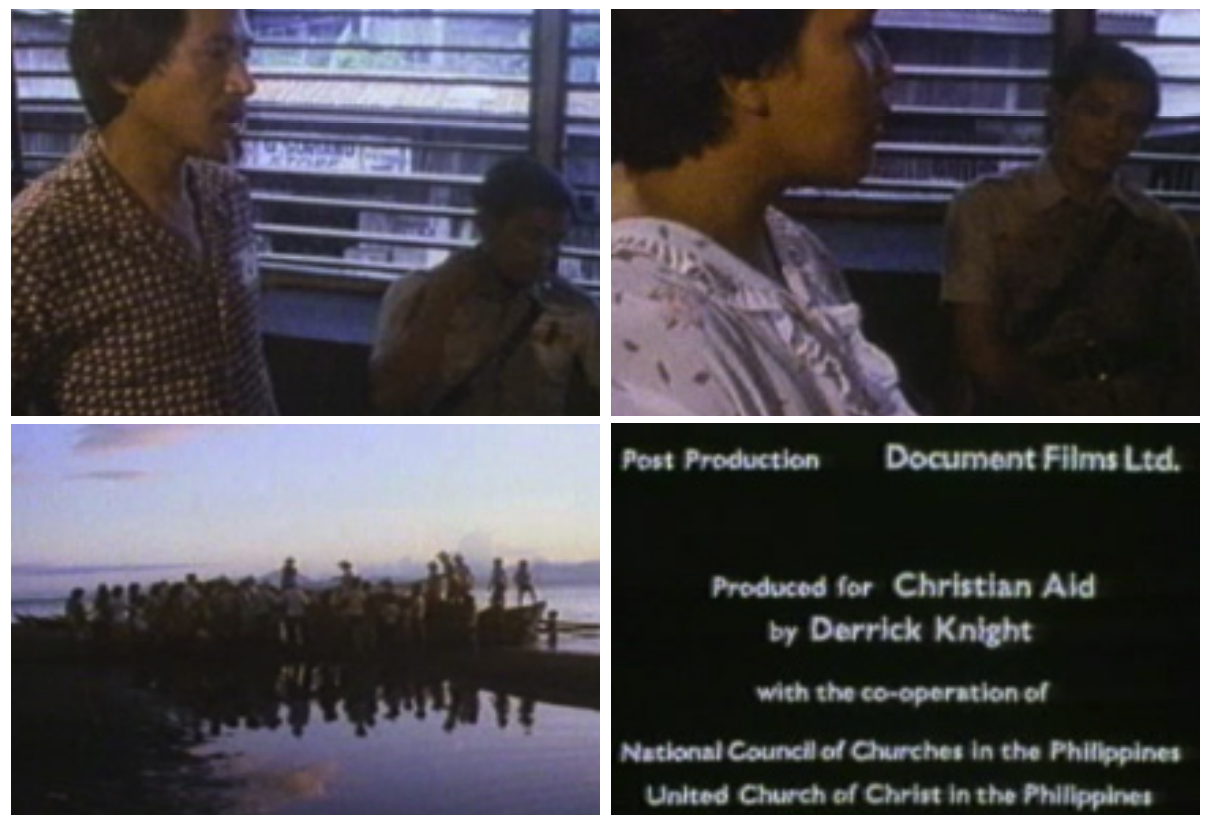

Larawan 5-8. Mula sa snapshot sa No Time for Crying (AsiaVisions, Christian Aid, \& Tiongson, 1986): Mga imahen ng kolektibong aksiyon ng mga residente ng Agusan del Norte sa Munisipalidad ng Magallanes, kasama ang bahagi ng credits ng pelikula sa dulo nito.

\section{Edjop (1986, 54 minutos, orihinal sa U-matic)}

Isang dokumentaryo ng AlterHorizons ang Edjop (Edgar Jopson Foundation \& AlterHorizons, 1986) tungkol sa buhay at pakikibaka ng lider-estudyante na si Edgar Jopson, kilala bilang si Edjop, na pinaslang noong Setyembre 1982 sa edad na 34 taong gulang.

Pinatingkad sa dokumentaryo ang buhay ng isang intelektuwal na nahubog ng kaniyang panahon at nagpasiyang paglingkuran ang mamamayang Filipino. Makikita ang taimtin na pagsandig ni Jopson sa lakas ng masa bilang puwersa ng pagbabagong panlipunan sa bungad pa lamang na awiting nagpapatingkad ng papel at pagkilos ng karaniwang tao para baguhin ang lipunang Filipino.

Nababaybay sa dokumentaryo ang naging buhay niya mula pagiging lider-estudyante ng moderate wing ng National Union of Students of the Philippines (NUSP) hanggang sa pag-igting ng kaniyang pakikisangkot nang maganap ang pagsunog sa dalawang baryo sa Bantay, Ilocos Sur dahil sa bangayan ng dalawang lokal na politiko. Malaking epekto rin ang naidulot sa kaniya ng pagkakataon na mabisita niya kasama ng mga iba pang estudyante ang Tsina na sa panahong ito'y nasa pamumuno ni Mao Zedong.

Nang siya'y nag-aaral ng abogasya sa UP College of Law, nasa hanay na ng kilusang manggagawa si Jopson bilang organisador hanggang tuluyan siyang 


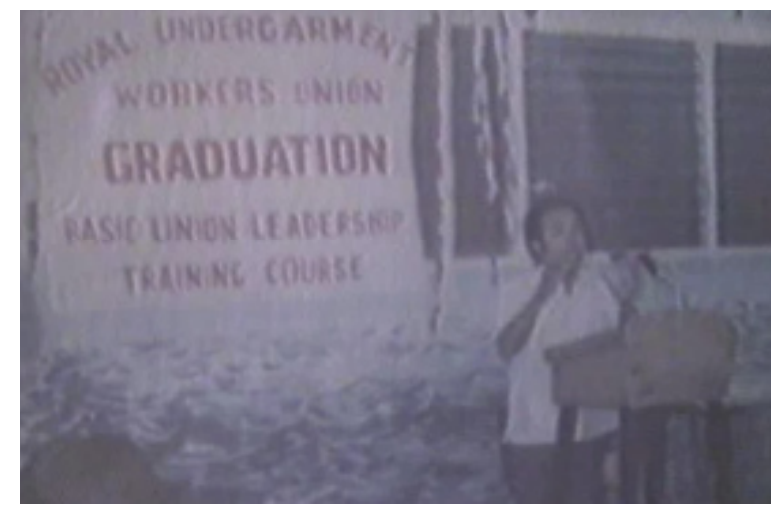

Larawan 9. Snapshot mula sa Edjop (Edgar Jopson Foundation \& AlterHorizons, 1986), larawan kuha mula sa isang Basic Union Leadership Training Course ng isang kasaping unyon ng GATCORD.

buong panahong kumilos sa hanay nito. Pinatampok sa mga panahong ito ang malaking papel niya sa mga pagkilos na kinalaban ang takot na hinasik ng Batas Militar, lalo na sa pagtatayo ng mga pederasyon ng mga unyon tulad ng National Union of Garment Textile Cordage and General Workers of the Philippines (GATCORD).

Makikita rin ang higpit at matalik na relasyong nabuo ni Jopson sa lidermanggagawang si Ka Felicing

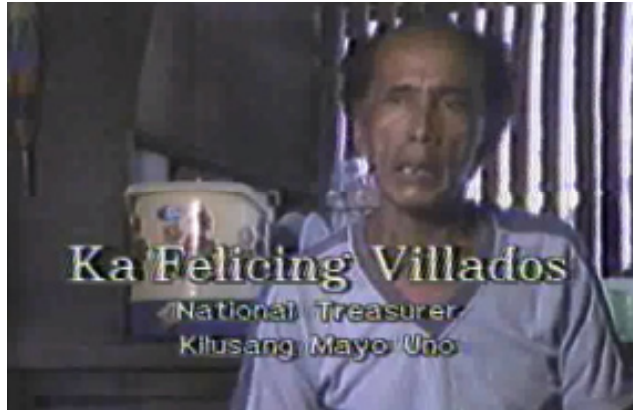

Larawan 10. Snapshot mula sa Edjop (Edgar Jopson Foundation \& AlterHorizons, 1986) ng panayam kay Ka Felicing Viilados ng KMU.
Villados, naging presidente ng unyon ng Elizalde Rope (C. Villados-Valdellon, personal na komunikasyon, 6 April 2016) at kinalaunan tumayong pambansang ingat-yaman ng Kilusang Mayo Uno (KMU). Tiniyak ni Jopson na sa kaniyang simpleng kasal, isang lidermangagawa ang maging prinsipal na isponsor nila, sa katauhan ni Villados.

Isinalaysay din sa pelikula na nadakip si Jopson noong Hunyo 1979 at sa isang voice recording sa kaniyang anak na si Nonoy, muling ipinamalas ang malaking pananampalataya ni Jopson sa masa. Sinabi niya sa pag-alay ng isang awitin sa anak: "Iyang kantang iyan... noong pinahirapan ako ng kaaway sa loob, sinuntok nila, tapos kinulong sa kuwartong madilim, tinali... kinakanta ko lang iyan para huwag nila akong matakot sa kanila. Kaya nakakatulong din kung alam natin ang mga kanta ng masa, nakakapagbigay sa atin ng tapang at lakas ng loob..." (Edgar Jopson Foundation \& AlterHorizons, 1986).

Malinaw ring isinalaysay sa dokumentaryo ang malaking papel ni Jopson sa pagkakatatag ng National Democratic Front of the Philippines (NDFP) at nang sumapi sa New People's Army (NPA), ang kaniyang ambag 
sa pakikibaka ng mamamayan sa Mindanao nang isinagawa niya ang preliminary social investigation (PSI) sa lugar. Kinikilalang malaking tulong ng nagawa ni Jopson sa pamumunong politiko-militar ng hukbong bayan at sistematikong paggampan ng gawain nito.

\section{Beyond the Walls of Prison (1987, 60 minuto, orihinal sa U-matic)}

Sa Beyond the Walls of Prison (AsiaVisions, Samahan ng mga Ex-Detainee Laban sa Detensiyon at Aresto [SELDA], \& Tiongson, 1987), inilantad ng videodokumentaryo ng AsiaVisions ang masalimuot na kalagayan ng mga detenidong politikal sa panahon ng diktadurang Marcos. Itinuring ng dokumentaryo ang mga detenidong politikal bilang mga buháy na halimbawa ng sistematikong paglabag ng karapatang pantao sa bansa.

Sa tindi ng presyur sa pandaigdigang saklaw at bunga ng People Power, daan-daang mga detenidong politikal ang pinalaya sa panahon ng gobyernong Aquino ngunit ipinakita na walang seryosong programa ang gobyerno para sa reintegrasyon ng mga dating detenidong politikal.

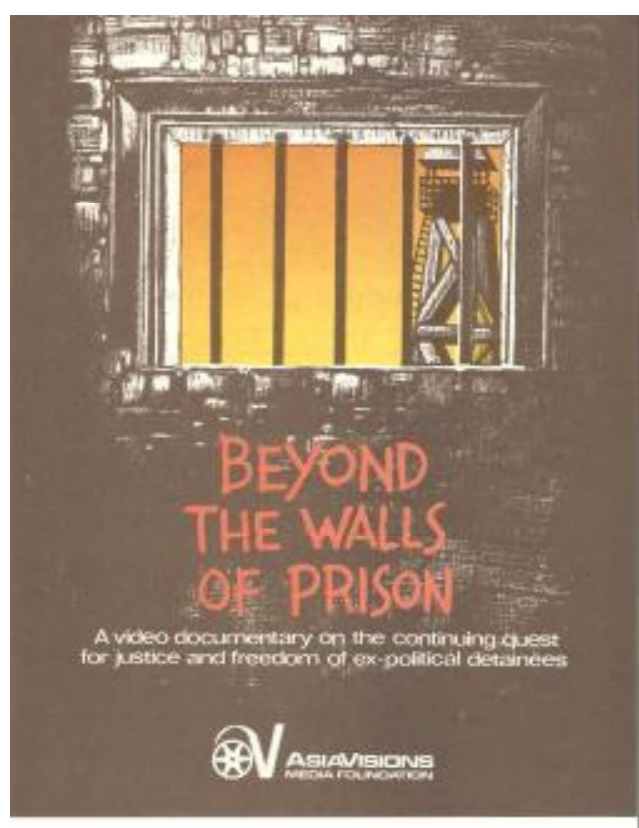

Larawan 11. Kopya ng one sheet (AsiaVisions one sheets, n.d.) ng Beyond the Walls of Prison (AsiaVisions, SELDA, \& Tiongson, 1987).

Kaya ipinakita sa pelikula ang pagsisikap ng mismong mga dating detenidong politikal na tulungan ang kanilang sarili, sa pagtatayo ng Samahan ng mga Ex-Detainee Laban sa Detensiyon at Aresto (SELDA) at sa pagsuporta ng iba pang organisasyon sa kanila tulad ng pagtatayo ng Medical Action Group (MAG) at Philippine Action Concerning Torture (PACT). Nagsimula ang dokumentaryo, halimbawa, sa pagtitipon ng lahat ng mga dating detenidong politikal na sa layuning pataasin at patatagin ang kanilang mapanlabang diwa.

Layunin ng dokumentaryo na malaman ng manonood ang mga kuwento ng pakikihamok ng mga dating detenido. Pinatampok ang katotohanang matinding kahirapan ng kanilang kalagayan kahit nakalaya na. Halimbawa rito, ang patuloy na pag-alala sa kanilang malupit na karanasan sa kamay ng mga sundalo, lalo na sa mga nakaranas mismo ng seksuwal na pang- 
aabuso at panggagahasa. Tampok dito ang mga karanasan nina Adora Faye de Vera at Hilda Narcisa. Kinakailangan nila ng psychological treatment para matulungan silang makaigpaw nang tuluyan.
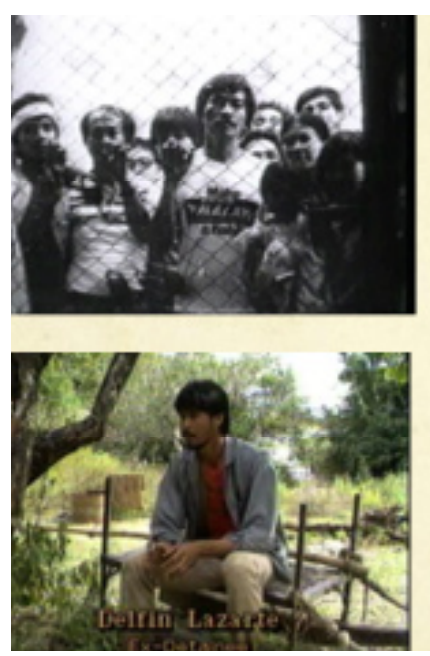
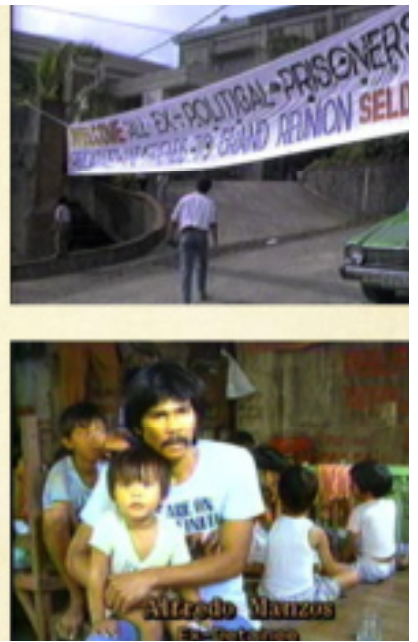

Larawan 12-15. Mga snapshot mula sa Beyond the Walls of Prison (AsiaVisions, SELDA, \& Tiongson, 1987). Ilan sa mga detenidong politikal sa panahon ng diktadurang Marcos tulad nina Satur Ocampo (taas-kaliwa), Delfin Lazarte (baba-kaliwa), at Alfredo Manzos (babakanan).

Makikita sa video-dokumentaryo ang tibay ng paninindigan ng mga naging detenidong politikal sa kanilang pagsisikap na manaig sa pananakit sa kanila. Nalabanan nila ang panghihina ng loob at hindi bumigay sa detensiyon at tortyur. Ibinahagi ni Adora Faye de Vera na minsan niyang tinangkang magpakamatay ngunit napagtanto niya na ito ang mismong nais ng mga pasista kaya hindi niya itinuloy ang pagpapakamatay. Sinabi niya sa sariling magpatagalan sila ng mga militar.

Itinampok din sa video ang katotohanan na ang mga magsasaka at manggagawa ang bulto ng naging detenidong politikal. Itinampok sina Delfin Lazarte, isang magsasakang nakaranas ng pagmamalupit sa kamay ng militar noong 1975 at si Ireneo Pring, kapuwa-magsasaka, na nakalaya lamang matapos magbayad ng piyansa ang kaniyang pamilya matapos magbenta ng kanilang munting lupang sinasaka. Itinampok din ang lider manggagawa na si Alfredo Manzos, isang manggagawang napatapos sa pagaaral ang kaniyang sarili sa pagsabay na pag-aaral sa gabi at pagtatrabaho sa pabrika sa araw. Dinakip si Manzos kasama ang 20 pang lider unyonista noong 1982 at lumaya noong 1985. Sa kaniyang paglaya, hindi na siya muling tinanggap ng pabrika. Naglunsad sila ng mga kapuwa-manggagawa ng welga noong Mayo 1985.

Itinampok din sa dokumentaryo si Alfonso Sabilano, 14-taong nakulong, at ang kaniyang patuloy na paghahanap ng katarungan sa kanilang sinapit. Kaniyang inihayag na hindi niya hahayaang malimot ang kaniyang naging karanasan hanggang walang natatamong katarungan at 
kaniyang ipapatanto sa kaniyang anak ang kaniyang dinanas bilang paalala na hanggang hindi nagbabago ang sistema, maaring maulit ang ganoong malupit na karanasan.

Ipinakita rin sa video ang kasalatan sa mga abogadong hahawak sa mga kaso ng detenidong politikal dahil sa dami ng inaaresto sa panahon ng diktadurang Marcos at nagpatuloy sa ilalim ng gobyernong Aquino (pagaresto kay Rodolfo Salas, halimbawa).

Naibahagi rin sa video ang karanasan ni Chino Roces, isa sa mga unang ipinakulong ng diktadurang Marcos. Itinuring niyang isang malaking concentration camp ang bansa na mas masahol pa sa mismong piitan kung saan siya nakulong.

Ipinakita rin sa video ang maagang pagkasira ng imahen ng gobyernong Aquino sa asasinasyon sa lider-mangagawang si Rolando Olalia ng KMU at kaniyang drayber na si Leonor Alay-ay. Naisadokumento rin ng video ang naganap na masaker sa Namulandayan, Lupao, Nueva Ecija noong 10 Pebrero 1987. Isang bangkay ng matandang babae ang ipinakita na ililibing, bukod sa kalagayan ng baryo matapos ang masaker at pagsunog na ginawa ng mga militar. Hindi na nagbigay pa ng detalye ngunit sa mga nakaaalam, magkasamang gálit at lungkot ang madadarama sa imahen ng matandang ililibing lalo na ang sinasabi ng mga militar ay NPA ang lahat ng kanilang napatay sa Namulandayan. Wala ni isa sa kanila ay kasapi ng NPA.

Nakapagbahagi rin ng kaniyang pananaw ang isa ring detenidong politikal na si Francisco Nemenzo. Sa dokumentaryo, isinaad ni Nemenzo, ang nangyari kay Olalia ay maaaring pagsisimula ng summary execution sa paraang wala nang pang-aaresto na tulad ng naganap noong Batas Militar kundi tuwiran na lamang na pagpapatay sa mga kritiko ng gobyerno.

Higit sa lahat, ipinakita sa video ang direktang epekto ng detensiyong politikal at iba pang paglabag sa karapatang pantao sa mga bata, maging direkta o di-direkta mang biktima ng paglabag. Ipinakita ang pagsisikap sa pagtatayo ng Children's Rehabilitation Center (CRC) para matulungan ang mga bata.

Ang lahat ng mga nailantad ng video ay dudulo sa pagkadesmaya at pagkawala ng pag-asa sa kakapalit lamang sa diktadura na gobyernong Aquino. Inilantad ng video ang kawalang kapangyarihan ng komisyon para sa karapatang pantao na usigin ang mga maysala at lumabag sa karapatang pantao.

\section{Mendiola Massacre (1987, 20 minuto, orihinal sa U-matic)}

Sa Mendiola Massacre (AsiaVisions, 1987b), makikitang muli si Delfin Lazarte ang magsasakang naging bilanggong politikal at naging bahagi ng video na Beyond the Walls of Prison. Sa pagsadokumento ng AsiaVisions paglahok ni Delfin sa isang pagkilos ng magsasaka noong 21-22 Enero 1987, 


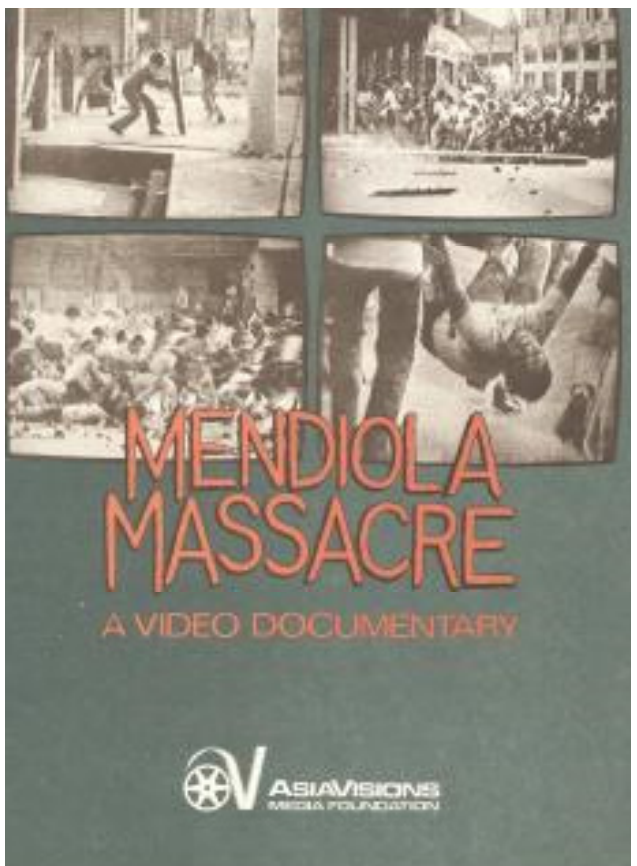

Larawan 16. Kopya ng one sheet (AsiaVisions one sheets, n.d.) ng Mendiola Massacre (AsiaVisions, 1987b).. naisadokumento ng AsiaVisions ang naganap na masaker sa Mendiola noong Enero 22 na mayroong 13 magsasaka ang nasawi matapos magpakawala ng sunod-sunod na putok ang militar at pulis sa mga nagpoprotestang magsasaka sa araw na iyon.

Naisadokumento ng video ang rali na nagsimula sa isang programa sa Liwasang Bonifacio, ang komprontasyon sa pagitan ng opisyal sa gobyernong Aquino at ng lider-magsasaka tungkol sa mgakahingian ng mga magsasaka, at ilang lider magsasakang nagsalita sa rali kaugnay ng isyung dala-dala nila sa araw na iyon. Sa panahong nabanggit, ang noo'y pangulo si Ginang Aquino ay sinabing nasa Cabanatuan at naglalako ng iminumungkahing Saligang Batas (na iraratipika pa

lamang). Sa araw ng pagkilos na iyon ng magsasaka, ang usapin sa kanilang karapatan sa lupang sinasaka ang nagtulak sa kanila upang magprotesta at tumungo sa Mendiola.

Naisadokumento ng AsiaVisions sa video na ito ang ilang pangyayari patungong Mendiola, ang mga sundalo't pulis na nakaharang upang pigilan ang martsa papuntang Mendiola hanggang sa gitgitan sa pagitan ng mga humarang at mga nagmamartsang magsasaka tungo sa mga huling segundo bago ang sunod-sunod na putok na pumatay nga ng 13 magsasaka.

\section{Pagpapanatili ng kolektibong alaalang nakaimbak sa mga pelikulang politikal: Pagpapanatiling mga pamanang dokumentaryo}

Ayon kay Ray Edmondson (2004), isang karapatang pantao ang pagkakaroon ng identidad at kasama sa karapatang ito ang karapatan sa akses sa mga pamanang dokumentaryo (documentary heritage), kasama ang pamanang awdyo-biswal, ng kaniyang bansa. Kasama rito ang karapatang malaman na ang mga ito ay umiiral at kung saan mahahanap ang mga ito. Itinuturing na dokumento ang anumang nagrerekord o nagdodokumento ng isang bagay na 
may sadyang layong intelektuwal. Ang pamanang dokumentaryo ay anumang bagay na magagalaw; binubuo ng mga signos/koda, tunog at/o imahen; mapepreserba (ang pinaglalagyan ay di-buháy); mapaparami at maililipat; at produkto ng sinadyang proseso ng pagdodokumento ("moveable;" "made up of sign/ codes, sounds and/or images;" "preservable [the carriers are non-living];" "reproducible and migratable;" "the product of a deliberate documenting process") (Edmondson, 2002, p. 8).

May itinuturing na dalawang komponent ang pamanang dokumentaryo: ang nilalamang impormasyon ("information content") at pinaglalagyan ("carrier"), kapuwa parehong importante ang mga ito bilang bahaging mgaalaala (Edmondson, 2002, p. 8).

Kasama sa mga halimbawa ang mga aytem na awdyobiswal tulad ng pelikula, disc, at teyp-na maaaring narekord sa analog o digital na format, at sa pamamagitan ng mekanikal, elektroniko, o anumang paraan (Edmondson, 2002).

Sa ganitong kalagayan, isang kongkretong hamon ang patuloy na pangangalaga sa koleksiyon ng AsiaVisions at lahat ng likhang-sining ng mga politikal na kolektibong pampelikula. Kagyat at pangmatagalang usapin itong dapat tugunan para maseguro ang pag-iral ng bahagi ng kasaysayan na naidokumento ng AsiaVisions at mga katuwang at kasunod na politikal na kolektibong pampelikula.
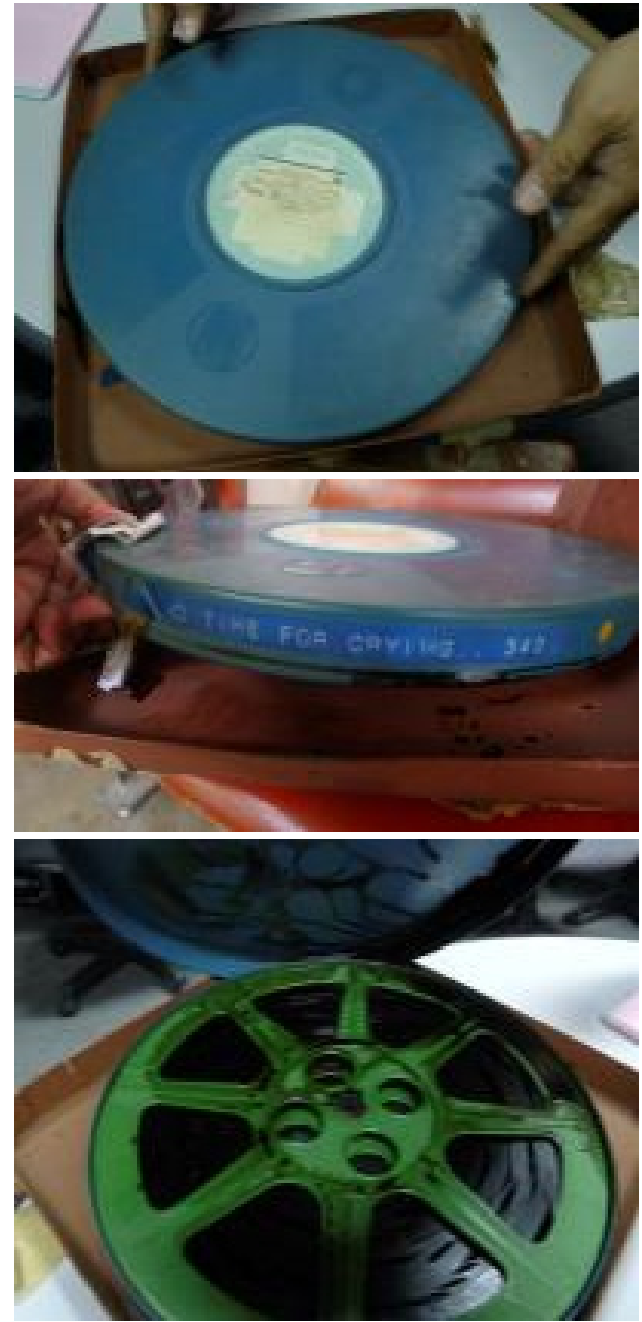

Larawan 17-19. Dokumentasyon ng isinagawang film inspection ng mananaliksik-manunulat sa tanging orihinal na kopya pelikulang No Time for Crying na nilikha gamit ang $16 \mathrm{~mm}$ film. Isinagawa ang inspeksiyon noong 11 Enero 2014 sa superbisyon ng mga propesyonal na artsibista ng National Film Archives of the Philippines (NFAP) (ngayo'y tinatawag nang Philippine Film Archive o PFA). 
Gayumpaman, napakalaking hamon at pagsubok ang pagtitiyak ng mga alaala, nasa pisikal o mental mang anyo. Sa panayam ${ }^{12}$ ni Ilagan sa First National Conference on Alternative Media na naganap sa Kolehiyo ng Pangmadlang Komunikasyon sa UP Diliman noong 10-11 Oktubre 2014, sinabi niya habang nililinaw ang saklaw ng kaniyang pagbabalik-tanaw sa nakaraan:

Hindi ko sasaklawin ang kabuoan ng pagkilos ng AlterHorizons at AsiaVisions. Unang-una, kailangang saliksikin ang mga bagay-bagay upang mangyari ito. Mabuti na lamang at mayroong gaya ng pagsisikap ngayon ni Prof. Rose Roque sa gawaing ito, na pinagkunan ko ng datos na nawaglit na sa aking isip. Alam po ninyo, kahit naging bahagi ako ng AsiaVisions, kinakalawang na rin ang aking memorya. (p. 2)

Sa bahagi ng custodian ng koleksiyong awdyo-biswal ng AsiaVisions, ilan pa lamang sa mga nasa koleksiyon ng AsiaVisions ang nailipat sa digital na format, hindi nila alam kung nagdikit-dikit na mga mahigit 1,000 teyp na nasa kanilang opisina. "On and off" na pinoproyekto nila ang digitization ng koleksiyon. Kaya lamang dahil malaki rin ang halaga at wala rin silang rekurso para doon, hindi pa napagpapatuloy ang proyekto, ayon sa dating executive director ng IBON na si Jaz Lumang (personal na komunikasyon, 22 Pebrero 2012).

Ayon pa sa kaniya, nagkaroon sila ng pana-panahong imbentaryo ng koleksiyon, ngunit walang kawani na eksklusibong nakatutok sa gawaing kaugnay ng pangangalaga ng koleksiyon. Nakaatang ito sa mga staff sa administrasyon.

Sumailalim sa receivership ng IBON Foundation ang AsiaVisions, kasama ang kabuoan ng audio-visual collection nito, noong mga taong 1998 hanggang 1999 (R. M. Papag, personal na komunikasyon, Marso 2012). Nang ipinasa sa IBON ang koleksiyon, tinuruan ang staff ng mga "basic" na hakbang para mapangalagaan ang mga teyp tulad ng bilin na pana-panahong paandarin ang mga teyp para huwag magdikit-dikit. Pero nasira ang ilan sa mga makina, mula sa tatlong U-matic player, halimbawa, na nauwi sa dalawa na naipagawa na lang din sa tulong ng mananaliksikmanunulat. Mayorya ng laman ng audio-visual collection ng AsiaVisions ay mga magnetic tape (U-matic, VHS, at Betamax). Ang natitirang pelikulang $16 \mathrm{~mm}$ na napasama sa koleksiyon ay sira na dahil sa vinegar syndrome. Ito ang tawag sa pagkatunaw ng film emulsion na nagreresulta ng pagkabura o pagkawala ng mga imahen ng pelikula. Kapag nangyari ito, wala nang maisasalba sa pelikula dahil wala nang mga imaheng mapoprojek mula sa negatibo. 
Para kay Lumang (personal na komunikasyon, 22 Pebrero 2012), ${ }^{13}$ pinakamahalaga sa kaniya ang pagsalba sa lahat ng maaring maisalba pa sa koleksiyon dahil lubos na nakapanghihinayang ang mga ito. Malaking hamon ang pagsasalba lalo na't wala pang sistema ng retrieval sa koleksiyon at nalagak lamang.

Naibukas ang pagkakataon sa kanila noong huling bahagi ng 2012 na makaugnayan ang noo'y head archivist ng National Film Archives of the Philippines (NFAP) na si Benedict "Bono" Olgado. Noon pa ma'y nagpahayag na si Olgado ng kahandaang pumunta sa opisina ng IBON upang makita ang buong koleksiyon para sa appraisal ng mga ito. May ilan ding inisyatiba pang sinisikap na gawin ang IBON kaugnay ng digitization ng koleksiyon at kabuoang hakbang para sa pangangalaga ng artsibo. Sa ngayon, bilang propesor sa School and Library and Information Sciences ng UP (UP SLIS) partikular sa arkaybing, pinamumunuan ni Olgado sa isinasagawang assessment ng koleksiyong AsiaVisions, sa mahigpit na pakikipagtulungan ng mananaliksik-manunulat at ng kasalukuyang executive director ng IBON na si Sonny Africa, para mailatag ang mas komprehensibo at matagalang plano sa pagprepreserba at proyektong digitization ng mga ito.

Isang malaking hakbang pasulong ang pagpili sa AsiaVisions Audio Visual Collection para maging kauna-unahang beneficiary ng Community Archiving Workshop(CAW) ng South East Asia Pacific Audio Visual Archives Association (SEAPAVAA) noong Abril 2017 nang idinaos din sa bansa ang internasyonal na kumperensiya ng SEAPAVAA (Community Archiving Workshop Manila Committee, 2017). Bahagi ng CAW Core Committee sina Mona Jimenez ng Moving Image Archiving and Preservation ng New York University (NYU-MIAP), Yvonne Ng ng WITNESS, at Marie Lascu, isang independent archivist. Sina $\mathrm{Ng}$ at Lascu ay nagtapos sa gradwadong masteral sa NYU-MIAP, kasama ni Olgado. Kinatawan ni Bono Olgado at ng mananaliksik-manunulat ang CAW Local/Manila Committee. Sa samasama at kolektibong pagtutulungang ito ng mga laybraryan, estudyante, filmmaker, AV archiving advocate, at iba pang volunteer, nakapagimbentaryo ang grupo ng $874 \mathrm{U}$-matic tapes sa pangangasiwa ni Olgado at ng mananaliksik-manunulat kasama ang mga kinatawan ng organisasyong UP ARCHONS na sina Greg Valerio at Liz Aldiano. Unang hakbang ang pagiimbentaryo para sa pangmatagalang layunin na mapreserba ang koleksiyon at kailangan para sa pagtukoy ng mga kailangang unahin sa oras na isagawa ang digitization ng mga kopya. Sa mas malawak na usapin, hindi pa tapos ang pagsasatitik ng kasaysayan ng Sineng Bayan.

Nasa kalikasan ng nagbabagong kasaysayan ng mamamayan na hanggang hindi nakakamit ang tunay na kalayaan at demokrasya, hindi hihinto ang paglaban at pagkilos. Hindi rin natatapos ang pag-iipon ng kolektibong 
mga alaalang nakapaloob sa mga likhang-sining. Hindi ito hihinto, kaya't kailangan na walang kapagurang gampanan ang pagtatala ng kasaysayan. Mahalaga ang gawaing ito hangga't may mamamayang hindi humihinto sa paggawa ng kasaysayan at hindi sumusuko na magkaroon ng pagbabago sa lipunan.

\section{Higit pa sa pagtatatala ng libong alaala}

Sabihin mang hindi ang kabuoan ng lahat ng pangyayari ng dekada 1980 ang naisadokumento ng dalawang grupo, ang pag-iral ng raw footage nito (na bahagi ng artsibong awdyo-biswal na nasa pangangalaga ng Ibon Foundation) ay makabuluhang bahagi ng kolektibong alaala ng mamamayang Filipino partikular ang bahagi ng kilusan at pakikibakang masa sa panahon at pagkatapos ng diktadurang Marcos.

Bukod sa mga nailathala ng IBON sa kanilang Video Catalog noong 2003 [sa brochure, mga may markang AV para sa kalakhang mga gawa ng AsiaVisions (pp. 2-9) at mga may markang AP, EU, NA, PH, IV para sa mga kopya ng pelikula at video, na noo'y pinaparenta ng Ibon Foundation, ng mga dokumentaryo mula sa

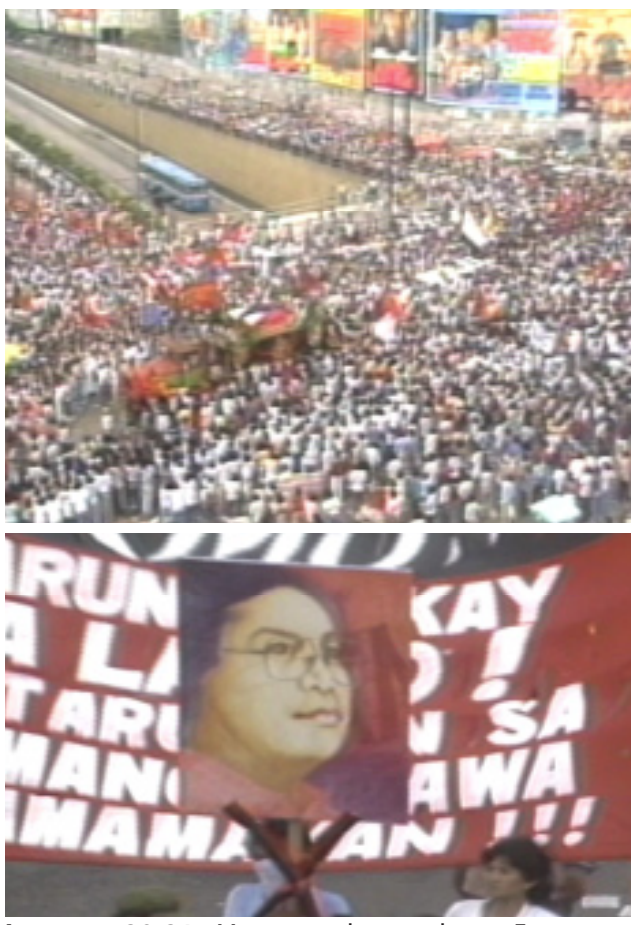

Larawan 20-21. Mga snapshot mula sa Fragments (1991). Ginamit sa video na ito ang footage ng martsalibing ng pinaslang na lider-manggagawang si $\mathrm{Ka}$ Lando Olalia ng Kilusang Mayo Uno. Asya at Pasipiko, Latin Amerika, Pilipinas, at iba pa (pp. 11-19)], mayroong substansiyal na bilang ng mga teyp na U-matic (U-matic at U-matic S) ng AsiaVisions ang wala sa listahan dahil kalakhan ay mga raw footage na pinagbatayan ng mga likha (batay sa imbentaryo ng buong koleksiyon na isinagawa mismo ng mananaliksik noong Abril hanggang Mayo 2012). Sa partikular, ang koleksiyong may markang UMAV at U2 ay binubuo ng 712 teyp (37 teyp na may marakang UMAV at 675 teyp na may marakang U2). Hindi pa kasama rito ang ibang mga teyp na U-matic din at iba pang mga teyp na Betamax at VHS (may mga markang AS, CC, $\mathrm{CM}, \mathrm{CN}$, EN, FE, U, U2P, UG P, at UK). Ayon kay Lumang, nasa iba't ibang antas ng storage ang artsibo. May 
ilan na silang nailipat sa anyong digital (J. Lumang, personal na komunikasyon, 22 Pebrero 1012).

Isa sa tampok na orihinal na footage na kuha ng AsiaVisions ng Lakbayan noong 1984. Produkto ng nasabing dokumentasyon ang Lakbayan '84 (AsiaVisions, 1984). Isa itong dokumentasyon ng Lakad ng Bayan para sa Kalayaan, isang mahabang martsa-pagkilos ng mga magsasaka, manggagawa, estudyante, propesyonal, taongsimbahan, mga manggagawang pangkultura na naglakbay ng isang linggo (mula Marso 1 hanggang Marso 7) mula Concepcion, Tarlac sa Hilaga ng Luzon at Lungsod San Pablo, Laguna sa Timog ng Luzon upang ipakita ang matibay na paninindigan laban sa eleksiyon noong Mayo 14 ng taong nabanggit. Boykot

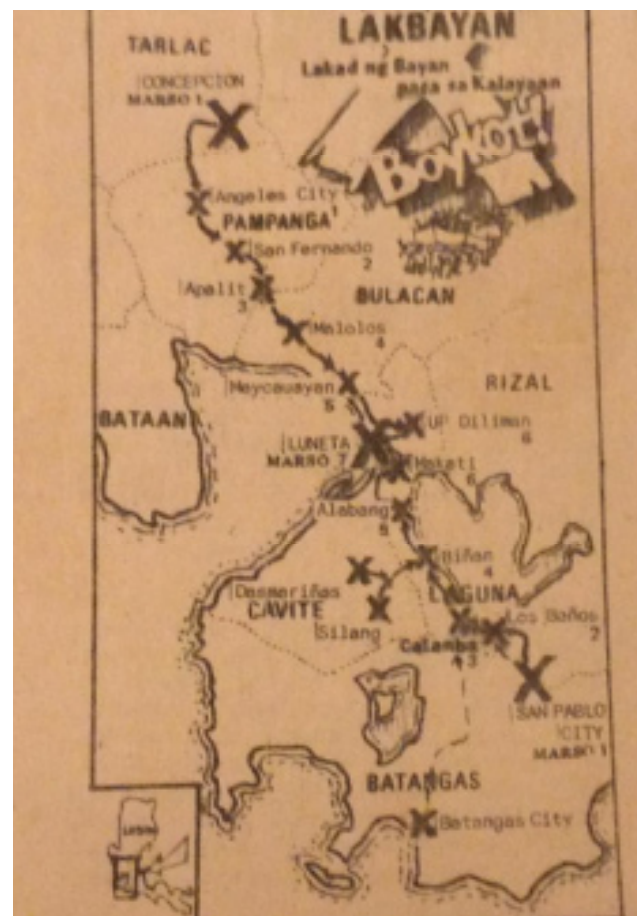

Larawan 22. Ruta ng Lakbayan na naganap mula Marso 1 hanggang Marso 7, 1984. Nagtagpo ang mga nagprotesta sa Luneta Grandstand noong Marso 7 (Ang Bayan, Marso 1984). ang panawagan sa pagkilos na nabanggit (N.G. Tiongson, 1984; Ang Bayan, 1984).

Nasa konteksto ang pagboykot ng halalan para sa 183 puwesto sa Batasang Pambasa noong 1984 dahil sa laganap na paniniwala, lalo ng oposisyon, na dadayain ni dating Pangulong Marcos ang resulta ng halalan (Lohr, 1984). Sa pangkalahatan at sa pangunahin, kadalasan ay dokumentasyon ng mga maiinit na pambansang isyu at mga pangunahing kampanya ang gawain ng AsiaVisions.

Isa rin sa tampok sa mga orihinal na footage ${ }^{14}$ ng AsiaVisions ang Kampo ng Bayan, isang taunang pagkilos ng mga magsasaka sa pangunguna ng Kilusang Magbubukid ng Pilipinas (KMP), na naganap noong 21-22 Enero 1987 na humantong sa tinatawag na ngayong Mendiola Massacre na naganap noong ika-22 Enero (AsiaVisions 1987a).

Kasama rin ang orihinal na footage ang naganap na martsa-libing noong naunang taon (20 Nobyembre 1986) para sa napaslang ang lidermanggagawa na si Rolando Olalia at kaniyang drayber na si Leonor Alay-ay (napaslang noong 13 Nobyembre 1986) (AsiaVisions, 1986). 


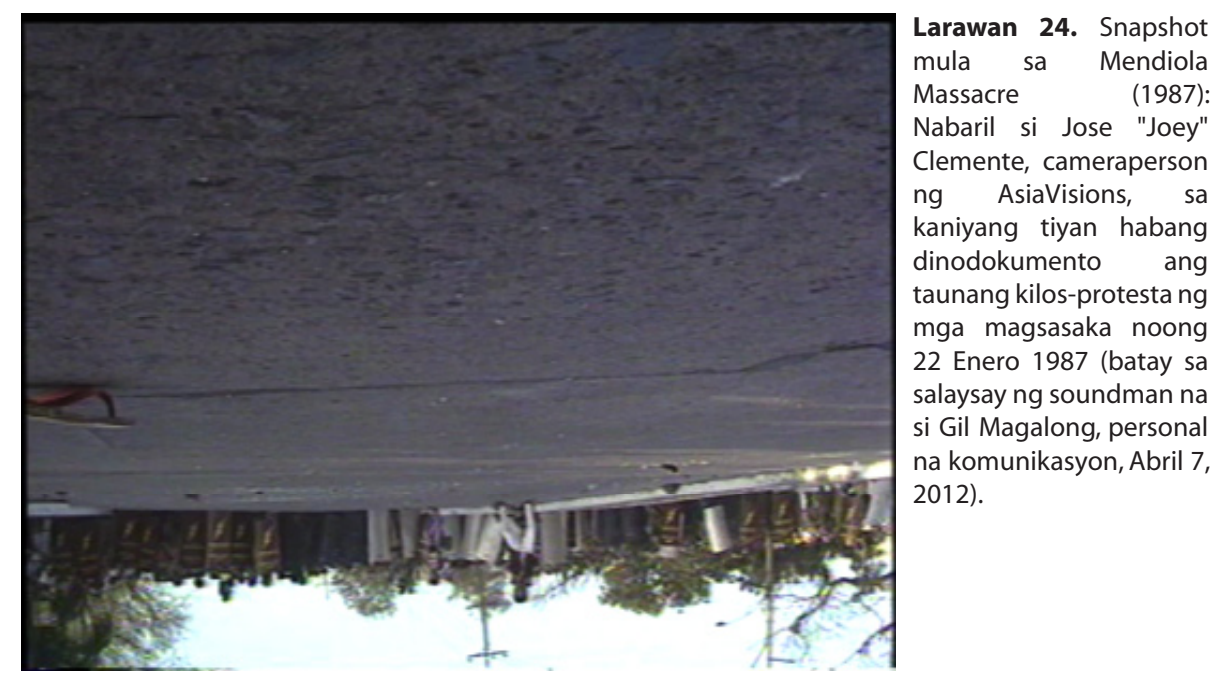

Kasama rin sa footage ng AsiaVisions ang paglahok ng Partido ng Bayan sa eleksiyong senatoryal noong Mayo 1987 (partikular ang mga kuha sa Miting de Avance ng grupo sa Plaza Miranda) (AsiaVisions, 1987c).

Mayroon ding orihinal na footage din ang AsiaVisions ng mga pangyayari kaugnay ng pagpatay sa lider ng Bagong Alyansang Makabayan na si Lean Alejandro noong 1987 (footage ng burol, martsa-libing, at mga pagkilos bilang pagkondena sa naganap na pamamaslang tulad ng transportation strike sa Cubao) (AsiaVisions, 1987d).

Higit sa lahat, masasabing ang mismong karanasan ng grupo ay matuturing at mismong bahagi na ng kasaysayang dapat itala/nakatala at malaki ang papel ng artsibo para magawa ito. Isang tampok na karanasan ng AsiaVisions ang nangyari sa Mendiola nang namasaker ang mga magsasaka at kasama sa nasugatan si Jose "Joey" Clemente, isa sa dalawang cameraperson ng AsiaVisions na noo'y nagdodokumento sa nagaganap na pagkilos (G. Magalong, personal na komunikasyon, 7 Abril 2012). Sa unang anibersaryo ng masaker, gumawa ng dokumentaryong Mendiola Masscre: A year after (AsiaVisions, 1988). Lingid sa kaalaman ng marami, pinangunahin ni Ishmael Bernal ang nasabing produksiyon, ayon kay Ariel Saturay (personal na komunikasyon, 15 Pebrero 2007).

Lalong-lalo na't bago ang pagsisikap ng pananaliksik na ito, wala pang pag-aaral na nagtala ng komprehensibong karanasan at kasaysayan ng AsiaVisions bilang isang organisasyon, ayon kay Ilagan (personal na komunikasyon, 12 Marso 2012. Posibilidad na dahil sa dami at sa babád sa gawain, hindi nakasusulat o hindi nakapaglalaan ng mas maraming oras ang mga filmmaker sa pagsusulat ng kanilang sariling karanasan, ayon kay Luna Sicat-Cleto, na naging kabahagi sa ilang produksiyong awdyo-biswal 
para sa Socio-Pastoral Institute (SPI). Napakahirap na gawain ang magtipon sa komprehensibong paraan ng mga karanasan, kasama na ang teknikal na pagsisinop at pangongolekta ng mga likhang pelikula ng mga grupo, kaya't isang nanatili ito sa matagal na panahon bilang isang hamon na hindi kagyat na natugunan, ayon kay Bobby Roldan (personal na komunikasyon, Abril 17, 2012) ng AlterHorizons.

Hindi lang din sa loob ng mga artsibong awdyo-biswal naitala ang mismong karanasan ng AsiaVisions bilang nuno ng politikal na kolektibong pampelikula. Isang publikasyon sa partikular ang nagbigay-espasyo upang maibahagi ni L. Tiongson ang kaniyang karanasan bilang filmmaker. Sa isang aklat ni Isabel Enriquez Kenny, isang akademiko-awtor na umakda ng Making documentaries in the Philippines (1996/2005), naibahagi ang naging karanasan ng AsiaVisions sa paggawa ng No time for crying. Narito naitala ang bahagi ng kasaysayan (L. Tiongson sa sipi ni Kenny, 1996/2005):

The situation in the countryside was, to say, the least, very volatile... We stayed in Agusan del Sur where the NDCGuthrie plantation was known, known as "farm of fear," is located and where an armed paramilitary group called "Lost Command" is known to operate.... Because of the critical situation of the area, we anticipated some protest actions and other forms of confrontation to happen within our sixweek shooting schedule....

We did not have to wait to shoot what proved to be an important scene for our documentary. Upon our arrival, we learned that a popular local labor leader was jist shot dead in broad daylight outside his house. We were able to shoot the wake, the funeral march, which turned into a protest action and the highly emotional interment ritual at the local cemetery....

A spontaneous confrontation scene also happened early in our shoot. A victim of police abuse and torture, together with relatives and community leaders, confronted his torturer at the office of the local mayor and demanded that justice be served. This mayor was put on th espot, groping for a Solomonic solution to the problem. This scene showcased the growing empowerment of the people in the countryside.

Two other extreme cases capped our shooting in Mindanao. One was an unplanned interview with a stalwart of the Sagrada Corazon Senor, a religious fanatic group enlisted by the military to sow terror and to fight the 
communist insurgency. After a long and exhausting drive to his place in a remote village, our film group was put on edge by his unusual behavior. With piercing blood-shot eyes and a bolo on his hand, he did not utter a word in front of us for an hour or two, as if on a ritual trance. Concealing our fear, we situated ourselves near the window of his house, he acceded to a short interview but did not want to be photographed, warning that any camera taking his picture would damage itself irreparably. Realizing the futility of rational argument with him, I instead quietly instructed my cameraman to roll the camera secretly when our subject was not looking.... (pp. 118-119)

Imbakan din ng alaala ang aktuwal na mga indibidwal na bahagi at/o saksi sa mga pangyayaring ininugan ng Sineng Bayan. Malinaw sa alaala ni Deocampo (personal na komunikasyon, 13 Pebrero 2014) ang naging pagtanggap at pagtangkilik sa mga gawa ng AsiaVisions (partikular sa pagtanggap ng manonood sa mga pelikulang Arrogance of Power at No Time for Crying). Matapos ang People Power, inorganisa ni Deocampo ang Independent Film and Video Festival noong 23-28 Setyembre 1986 sa idinaos sa New Wave Cinema sa Araneta Center Cubao sa Lungsod Quezon. Dito ipinalabas ang Arrogance of Power noong 24 Setyembre 1986 (Miyerkoles) at inulit noong 27 Setyembre 1986 (Sabado) ang tinaguring premiere screening ng No Time for Crying noong 24 Setyembre 1986; sa kaparehong araw (Setyembre 24) naipalabas din ang Sabangan (8mm, 23 min.) nina Jose Cuaresma, Bedette Libres, et al. at Kaigorotan (video, 37 min.) ng AlterHorizons (premiere screening) (Independent Film and Video Festival, 1986).

Sa pagsasaliksik at pagsisikap ni Deocampo (personal na komunikasyon, 13 Pebrero 2014), nahanap niya ang mga pelikulang naisama sa nasabing festival. Ayon sa kaniya, napuno ang 700-upuang sinehan, puno hawak ang mga pulang watawat: “... puno ng leftists na puno ng... red flag-waving na mga audience nila. Talagang they delivered the audience also. Can testify talaga na napuno ang 700 -seater dito sa Wave Cinema, wala na iyan ngayon. Pero those of us who remember that...."

Ayon pa kay Deocampo (personal na komunikasyon, 13 Pebrero 2014):

Bawat alas-otso ng gabi, ginagawan ko sila ng panggabi na screening at pinupunô nila 'yan... pinapaskil ang... banners nila.... And that's why nakikiisa ako sa kanila kasi alternatibong-alternatibo ang audience nila. Usually kasi ang audience ko..., estudyante iyan... may mga NGO ako na iba... 
kababaihan. Pero ito, iba iyong... maaamoy mo sila, 'yung galing sa init..... Masa, 'yung totoong masa. So, ang gandaganda. Pero ang mga pulis, natataranta. Iniikutan ako agad niyan. Kailan ko siya ipinalabas? Pangalawang araw yata. So medyo pagkatapos iyon, binabantayan na kami. After that, pangalawang labas, medyo mainit na ang mata.

Makikita rito at maihahawig sa tinuturan ni Ilagan (2014, p. 3) na mass base bilang "isang sistema at estruktura ng distribution na nakasalalay sa daan-daan libong kasapian ng mga organisasyong masa, at sa milyonmilyong baseng suporta ng kilusan."

Ayon kay Libres (personal na komunikasyon, 7 Hunyo 2012), naging executive director ng Kodao, malinaw sa mga pagtatalang ito na ang mga kalakaran at karanasan ng produksiyon ng at resepsiyon sa mga likhang pelikula ng mga politikal na KP na kumakatawan sa Sineng Bayan ay hindi lamang kaugnay ng pagdodokumento ng kasaysayan kundi paggawa mismo ng kasaysayan. Ganito rin ang pananaw ni Eli Guieb (personal na komunikasyon, 13 Enero 2011), naging bahagi ng AlterHorizons at naging assistant director sa pelikulang Isang munting lupa (AsiaVisions, Cultural Center of the Philippines [CCP], \& Tiongson), na sila'y "hindi lamang nagdodokumento ng kasaysayan, gumagawa rin ng kasaysayan."

\section{Mga politikal na kolektibong pampelikula bilang bahagi ng malawak na kilusang masa: Sineng Bayan bilang politikal na sandata ng nakikibakang mamamayan}

Nakikita sa mga karanasan, kalakaran, at kasaysayan ng Sineng Bayan kung paano ginamit ang mga anyong kultural na pelikula at video bilang politikal na sandata ng nakikibakang mamamayan. Malinaw na ang Sineng Bayan ay nakalubog sa mga búhay at pakikibaka ng mga mamamayang Filipino. Malinaw sa bawat politikal na KP na sila't bahagi ng isang mas malawak na kilusan, ang kilusan ng mamamayan para sa pagbabago ng lipunang Filipino. Kaya't malinaw sa mga likhang pelikula at video ng mga politikal na KP kung ano ang nilalaman at kung para kanino inilalaan ang mga likha.

Isang sandatahang ideolohiko ang pelikula sa kamay ng mga politikal na kolektibong pampelikula dahil sa bisa at kapasidad nitong makapanghimok para sa direktang pakikilahok sa pagkilos ng mamamayan para sa pagbabagong panlipunan.

Masasabing kahawig ng mga karanasan ng Sineng Bayan ang nakita ni Teshome Gabriel (1979) sa karanasan ng mga Ikatlong Sinema sa ibang mga bansang itinuturing na bahagi ng Ikatlong Daigdig (sa kaniyang disertasyon na Third Cinema in Third World: The dynamics of style). Nakakahawig sa mga karanasan na ang mga ganitong uri ng pelikulang politikal, bahagi 
man ng Ikatlong Sinema o Sineng Bayan, ay hindi lamang maalam sa mga panlasang kultural at pangangailangang ideolohiko ng mga mamamayang mga kinakatawan ng mga ito kundi maging ng mga militansiya ng kanilang pakikibaka. Kaya nga't kapuwa sa Ikatlong Sinema at Sineng Bayan, ang pelikula ay armas.

Tulad ng pananaw ni Jose "Joey" Clemente (personal na komunikasyon, Mayo 8, 2012), cameraperson sa dokumentaryong Mendiola Massacre, ang kapangyarihan ng produksiyong video ay nasa kapasidad nito na mapakilos ang manonood. Dito posibleng masukat ang tagumpay o pagkabigo ng isang materyal. Mahalagang tanong na masagot sa panonood ng pelikula ay kung nakaramdam ba ang manonood ng pakikiisa sa mga inaapi at pinagsasamantalahan; kung sila ba ay may natutuhang bago sa kalagayan at pakikibaka ng mga masa; kung nakaramdam ba ng pagnanais na makisanib sa kanila?

Ang ganitong pagkiling ng Sineng Bayan para magsilbing kasangkapan at armas ang pelikula para sa pagbabago ay, malinaw na kaso, sa wika ni Raymond Williams (1977), ng seryosong pagpanig (komitment) para sa panlipunang realidad.

Kung ilalapat sa pelikula, ayon kay Williams (1977) sa pagsipi kay Sartre, na ang panitikan, kung hindi aalingawngaw sa lahat ng antas ng buhay ng tao at lipunan, ay walang silbi. Ito ang usapin ng komitment sa panitikan. Ang usapin ng komitment ay ang mulat na pagpanig o mulat na pagbago ng pagpanig.

Sa kaso ng mga politikal na kolektibong pampelikula, malinaw ang pagpanig kahit sa mga likha nitong dokumentaryo. Ayon kay Romulo Regalado (personal na komunikasyon, 24 Marso 2012), kasama ni Clemente bilang cameraperson sa dokumentaryong Mendiola Massacre, "kung tutuusin, walang video production na walang manipulasyon kahit man ito ay dokumentaryo sapagkat ang mga desisyong direktoryal ay manipulasyon na rin ng pagkalap at pagbuo ng dokumentasyon." Ngunit nililinaw niya na hindi ibig sabihin nito na ang isang video ay hindi na dokumentasyon ng realidad. Importante ay "walang ginawang manipulasyon ng dokumentasyon na hindi na sumasalamin o naayon sa mga pangyayari o sa realidad." Usapin ito ng epektibong pagpapakita ng mga tunay na mga nangyayari.

Ganito rin ang pananaw ni Marlene Francia (personal na komunikasyon, 9 Abril 2012):

Documentary filmmaking has been described as creative treatment of reality, of actuality and inherent in this definition is there is already "manipulation"..... (T)he act of filming a particular sequence and not another is already "manipulation". The fact that you chose this particular 
editing in-point and that particular out-point is already "manipulation." The fact of ascribing a certain image to convey a certain meaning to a certain piece of narration or voice over interview is already "manipulation."

Samantala, tinitingnan ito ni Clemente (personal na komunikasyon, 8 Mayo 2012) sa usapin ng pagbibigay lamang ng mga filmmaker ng puntode-bista, isang punto-de-bista ng mga naisasantabi sa lipunan ("You can only provide a point of view. Normally what we chose to show is the point of view of the people in the margins of society").

Realidad ito lalo na nagsisilbi para sa propaganda-edukasyon ang mga likhang-sining ng mga politikal na kolektibong pampelikula, tulad ng mga gawa ng AsiaVisions at AlterHorizons. Sa ganitong kalagayan, lagi't laging usapin ang "panganib" na iniisip na hatid ng mga likhang may politikal na adbokasiya. Gayumpaman, ang ulltimong layunin ng Sineng Bayan ay makapag-ambag sa pagkilos ng mamamayan para sa pagbabago. Tulad ng winika ni Ernst Fischer (1963):

Dapat mapanghawakan ng likhang sining ang tagapagtangkilik hindi sa pasibong pagkilala, kundi sa panghihikayat nitong maging makatuwiran na magbubunsod ng pagkilos at pagpapasiya (akin ang salin). The work of art must grip the audience not through passive identification but through an appeal to reason which demands action and decision. (p. 10)

Mulat na pagkilos at pagpapasya ng mga manonood ang sukatan ng bisa at silbi ng Sineng Bayan. Hindi magiging makabuluhan at makatuwiran ang pagkilos na bunga lang ng bulág na pagsunod sa "manipulasyon". Hindi ito maglilingkod sa isang kilusang naglalayon ng tunay na pagbabago sa lipunan, na magagawa lamang kung ang mga kasangkot ay may kamulatan, paninindigan, at dedikasyon upang sumuong nang di-matitinag at handang magsakripisyo sa kurso ng mahabang proseso ng panlipunang pagbabago.

Sa konteksto ng lipunang Filipino, makikita ang sining na may pinapanigan bunga ng tungkuling pinakamahalaga sa lahat, ang paglilingkod sa sambayanan. Makikita ang diwang ito sa mga nangyari sa unang mga taon ng pagkakatatag ng AsiaVisions at AlterHorizons. Unang naitatag ang AsiaVisions bilang NGO at isa sa tagapagtatag nito ang naging tagapagtatag din ng AlterHorizons bilang kooperatiba (J. Cuaresma, personal na komunikasyon, Marso-Abril 2012). Nangyari ito dahil sa paglitaw ng kaibahan sa estilo ng paggawa at pamamaraan ng organisasyon sa pagitan ng mga tagapagtatag ng AsiaVisions.

Ayon kay Jose Cuaresma (personal na komunikasyon, Marso-Abril, 
2012), noong 1986, nakumbinsi sila ni Bobby Roldan na magtayo ng sariling grupo na iba sa AsiaVisions. Mas ayon ito sa pananaw na di-limitado ang isang kooperatiba sa usapin ng paglaki ng mga magiging kasapi. Sa pananaw nila, hindi ito tulad ng isang NGO na limitado lamang ang maipapasapi rito (limitado sa mga staff lamang halimbawa). Matatandaan na sa panahong ito karerehistro pa lamang ng AsiaVisions sa SEC bilang NGO. Gayumpaman, hindi naging hadlang ito para patuloy na magtulungan ang dalawang pormasyon. Nagsilbing talent pool ng AsiaVisions sa ilan nitong produksiyon ang AlterHorizons (B. Roldan, personal na komunikasyon, Marso-Abril 2012; J. Cuaresma, personal na komunikasyon, Marso-Abril 2012). Nagbibigayan din sila ng mga kopya ng mga gawa ng bawat isa, kaya makikita halimbawa sa audio visual library ng AsiaVisions ang mga likha ng AlterHorizons. ${ }^{15}$ Malinaw ang layunin ng dalawa, iba mang estilo ng paggawa at anyo ng organisasyon, kapuwa sila pinagkakaisa ng layunin na paglingkuran ang sambayanang Filipino sa pamamagitan ng kanilang mga nililikhang pelikula. Nanatili silang magkatuwang sa gawaing kultural, sa partikular sa paggawa ng pelikula.

Bunga itong masikhay na paglilingkod ng tiyak na tungkulin ng pakikibaka para sa pambansang demokrasya laban sa imperyalismo, pyudalismo, at burukrata kapitalismo (Sison 1971/2001). Sa "Mensahe sa PAKSA ${ }^{16}$ Hinggil sa Mga tungkulin ng mga kadre sa larangan ng kultura," sinabi ni Jose Maria Sison (1971/2001) na kailangang "ihanda ang opinyong publiko" (pp. 335-336) upang magtagumpay ang pagbabago ng lipunan.

Kailangang magkaroon ng diwang mapanlaban ng mamamayan na pangunahing lalamanin ng "rebolusyonaryong kultura, na namumukod pero integral na bahagi ng kilusang masa" (Sison, 1971/2001, pp. 335-336).

Masasabing nagtutugma ang mga natukoy na kalakaran at karanasan ng mga politikal na kolektibong pampelikula at Sineng Bayan sa tinuran sa The New Mass Art and Literature ni Kris Montanez (1988) na taliwas sa kultura ng pasismo, ang kultura ng pambansang demokrasya ay naglilingkod sa masang Filipino sa pamamagitan ng pagpapakita ng kanilang kolektibong lakas. Ang nilalaman ng mga likhang bahagi ng kultura ng pambasang demokrasya ay mula sa mayamang rebolusyonaryong karanasan ng mga masang Filipino, na silang bumubuo rin ng pinakamaraming bilang na tagapagtanggap ng mga bagong sining at panitikan. Hindi lamang makabuluhan ang papel ng mga bagong sining at panitikan sa pakikibaka ng mga mamamayan, kundi bahagi na rin ang mga ito ng kanilang pang-araw-araw na buhay.

Sa mga karanasan ng mga politikal na KP, tunay ngang hindi sagka ang pera, kagamitan, at kasanayan sa paggamit ng pelikula bilang kasangkapan para sa pakikibaka, mapakultural man o politikal. Tunay ngang magagamit ng pelikula ang lahat ng kagalingang teknikal at teknolohiyang makakaya 
upang magamit para pagtibayin ang lahat ng usaping posibleng suungin. Mahahalitulad sa sinabi ni Gabriel (1979) na isang ngang pagkakamali na ituring na kagaspangan ang estilo ng ilang pelikula ng Sineng Bayan kung walang pagkilala sa mga materyal na salik at kondisyon na nagbunga ng gayon. Hindi nga kinis at teknik ng pelikulang Hollywood o mainstream man ang mga likha ng Sineng Bayan, natutugunan ng mga ito ang kinakailangang layunin. Sa mga tampok na likhang-sining, makikita sa imahen pa lamang na taglay ng mga ito, na naitatanghal ang tinig at karanasan ng nakikibakang mamamayang Filipino.

Malinawsakasaysayanngunangmga politikalnakolektibong pampelikula ang "pangunahing layuning gamitin ang video bilang kasangkapan ng kilusang masa" bilang isang matingkad na leksiyon kaugnay ng "pagsunggab sa bawat larangan at oportunidad na naririyan at dumarating upang isulong ang kilusang masa," ayon kay Ilagan (2014, p.2).

Tumutugma ito sa pananaw ni Deocampo (personal na komunikasyon, 13 Pebrero 2014) kaugnay ng pangangailangan ng pagpapaunlad ng "cinema of need" kontra sa "cinema of want." Ayon sa kaniya, kailangang tumugon sa pangangailangan ang paggawa ng pelikula. Makabuluhang tanong ito: Matatali lang ba sa pansarili ang dahilan? Sa ganitong punto, kinikilala ni Deocampo ang nagawa ng AsiaVisions sa paghanga sa grupo na gumawa ng pelikula dahil may pangangailangan at dapat mas magsikhay pa para sa layuning ito.

Mahalaga ang diskursong ito lalo na sa panahong ngayong ng digital filmmaking sa bansa.

Sa maagang yugto pa lamang ng pagsikad ng digital filmmaking, may pagpuna na sa komersiyal na mainstreaming ng mga pelikulang indie. Ayon kay Rolando Tolentino (2008a), nagreresulta ang ganitong tunguhin sa pangangailangan sa malawakang venue at market para sa mga pelikulang indie, partikular sa pagpasok ng mga ito sa mga komersiyal na sinehan at pag-abot sa mga manonood na makapagbabayad ng tiket ng sine. Hindi na matatangi ang pangyayaring ito lalo sa pag-usbong ng salitang "maindie" (mula sa mainstream at indie) na mahihinuha na tumutukoy, bilang kongkretong halimbawa, ng paggamit ng artistang mula sa mainstream ng pelikulang indie para sa mga role na hindi nagagampanan sa mainstream, sa isang banda (sa pagbanggit ni Jose Javier Reyes, isang direktor ng pelikula na naiulat ni Villena, 2012; Torre, 2013), at pagpasok sa mainstream ng mga indie talent at maging mga bagong direktor mula sa indie (Torre, 2013).

Malinaw na ang mga likha ng mga politikal na kolektibong pampelikula ay iba sa ano ba ang isang pelikulang indie. Malayong-malayo na ito sa panuntunan na unang inilatag ni Deocampo (1994) sa kaniyang akda sa CCP Encyclopedia na ang pelikulang independiyente ay pelikulang alternatibo na 
malinaw na hindi nakukupot sa komersiyal na filmmaking [na tinuturing na mainstream] at kung gayo'y umiiral sa labas nito. Sa yugtong ito, masasabing iba na ang esensiya ng pelikulang independiyente sa pelikulang alternatibo at hindi na mapag-iisa na lamang.

Isang mahalagang punto rin na "likas" na umusbong ang konsepto ng bayan sa hanay ng mga aktibista. Sa pagkaalaala ni N.G. Tiongson, sa panayam ng mananaliksik-manunulat, sa hanay ng mga Manunuri, ${ }^{17}$ hindi natalakay at lumutang ang konsepto ng People's Cinema. Ayon kay N.G. Tiongson (personal na komunikasyon, 11 Pebrero 2014), tiyak siyang sa hanay ng mga aktibista ito nadidiskurso. Sa panahong ito, ginagamit at laganap na ang konsepto ng "People's Theatre," ngunit hindi ang People's Cinema. Masasabing isang paliwanag dito, tulad ng nabanggit kanina ayon kay Luna Sicat-Cleto, na posibilidad na dahil sa dami at sa babad sa gawain, hindi nakasusulat o hindi nakapaglalaan ng mas maraming oras ang mga filmmaker sa pagsusulat ng kanilang sariling karanasan (L. Sicat-Cleto, personal na komunikasyon, 18 Mayo 2010).

Sinasalamin sa pag-aaral na ito ang pagsisikap na itala ang kasaysayan ng isang uri ng sinema na napapanghawakan ang mga manonood upang maging makatuwiran para makapagpasya at kumilos mula pagsalunga sa salita tungo sa pagsalunga sa pagkilos, sa gitna ng patuloy na paglaganap ng kasinungalingan sa bansa at sa papaigting na panunupil ng kalayaang sibil ng kasalukuyang rehimen.

Tinuran man ni Deocampo (1994) na marami sa mga independiyenteng likha na sumasalamin sa boses at tunay na imahen ng mamamayan ang nanatiling nakakubli, hindi na ito ang nangyayari. Sa pagtatalang ito ng karanasan, kalakaran, at politika ng Sineng Bayan, isang hakbang sa paglalantad, pagtatala, at pagtitiyak ng nagbabagong kasaysayan ng mamamayan ang naisasakatuparan.

Ang kaalaman sa kasaysayan at pag-unlad ng Sineng Bayan ay makatutulong sa pagsulong ng pelikula bilang isang mabisang sandatang pangkultura ng ating mamamayang Filipino sa ating mithiin para sa pambansang kalayaan at tunay na demokrasya. Ito ang kapangyarihang noon pa ma'y badya na ni Petronilo Daroy (1983), kasapi ng Manunuri ng Pelikulang Pilipino, na ang papel ng sinema sa ating pagpapakahulugan bilang Filipino at sa ating potensiyal bilang mamamayan.

Kakambal ng konseptong People's Cinema ang konseptong pagbibigaykapangyarihan sa mamamayan bilang manonood. Naniniwala si Roy Iglesias (personal na komunikasyon, 16 Enero 2012) na kayang baguhin ng manonood ang nilalaman ng sine (o telebisyon) kung nagkakaisa ang mga mamamayan bilang manonood. Nasa manonood ang kapangyarihan ng pagtangkilik at pagboykot ("patronage and boycott"). Tinitingnan niya 
na ang manonood, tulad at bilang konsyumer, ay mayroong ganitong kapangyarihan. Gayon lamang, hindi nakikita ng mga manonood ang panonood ng sine (o telebisyon) bilang isang gawaing may tiyak na layunin ("purposive activity"), na sa pagtangkilik o sa pagboykot, mababago ng manonood ang nilalaman ng sine (o telebisyon). Sa tingin ng manonood, pang-aliw at pampalipas-oras lamang ang panonood. Itinutulak ng ganitong pagtingin ang isang mulát na uri ng pagtangkilik sa pelikula at video-ang pagtangkilik sa isang sinema na magpapabago sa lipunan at sa ating panlasa. Sa ganitong kalagayan, ang pelikulang di-maganda at di-makatutulong sa ating pagkamulat ay hindi makakakuha ng pagtangkilik. Tinatawag ito ni Iglesias na aktibismo ng mga manonood ("audience activism"). Dagdag pa niya, hindi dapat "nasasayang" ang teknolohiya para lamang sa simpleng self-expression ng isang filmmaker. Sa tingin niya, mainam kung ang People's Cinema ang siyang alternatibong sinema. Dahil layon ng People's Cinema ang magmulat ng manonood, dapat na kalahok mismo ang manonood sa pagunlad ng sinema. Napakamakapangyarihan ng kamera bilang teknolohiya at dapat itong magamit para sa kapakanan at kamulatan ng manonood.

\section{Hinaharap na hamon}

Patuloy na hamon ang pagdodokumento ng mga karanasan ng mga politikal na kolektibong pampelikula at Sineng Bayan. Nariyan ang kahirapan ng nagbabagong teknolohiya sa paggawa ng pelikula at maging sa pag-akses ng mga ito. Ngunit hindi matatawaran ang kahalagahan na pahalagahan ang mga ito bilang pamanang dokumentaryo ng ating bayan.

Hindi matatawaran ang historikal-politikal na kabuluhan ng mga nasabing pamanang dokumentaryo bilang bahagi ng kolektibong alaala nating mga mamamayang Filipino. Malinaw ang nilalaman ng Sineng Bayan: karanasan at pakikibaka ng masang Filipino. Malinaw ang mga tagatanggap ng Sineng Bayan: ang naisasantabi at nakikibakang masang Filipino.

Patuloy ang kolektibong pagsalungat sa kasinungalingan at panunupil ng naghahari sa ating lipunan. Patunay ang patuloy na pag-iral at pagsulpot ng mga politikal na KP sa panahon ng digital filmmaking sa ganitong katunayan.

Higit sa lahat, kolektibo ang tradisyon ng mga politikal na kolektibong pampelikula dahil sa politikang hindi humihiwalay at kahit kailanman hindi mahihiwalay sa kilusan ng mamamayan.

Sa pangkalahatan, naipakita sa pananaliksik na sa pamamagitan ng pagbaybay sa mga karanasan, kalakaran, at mga likha ng Sineng Bayan, partikular ng mga likha ng AsiaVisions at AlterHorizons, makikita ang nagbabagong kasaysayan ng nasyon. Kumakatawan at nakapanig sa mga nasasantabing sektor sa lipunang Filipino ang mga politikal na KP. 
Sa marubdob na paggampan ng kanilang gawain, sa kanilang dokumentasyon ng kalagayan at pakikibaka ng mga mamamayan, sa pagpapaunlad ng mga kolektibong produksiyon, sa walang-kapagurang pagtatatala ng kasaysayan ng mamamayan, ang mga kasapi ng mga politikal na kolektibong pampelikula ay nakapag-ambag sa paglalantad ng kasinungalingang lumalaganap sa lipunang Filipino sa interes ng naghaharing Sistema.

Epektibong nagagawa ito dahil sa kolektibong paglikha ng mga KP ng mga pelikula, video, o AVP bilang isang organisasyong tumataliwas at nakikipagtunggali sa dominanteng kalakaran sa komersiyal na industriya ng paggawa ng pelikula.

Sa pananaliksik na ito, napatampok ang mahalagang usapin ng paggawa ng mga likhang pampelikula na tunay na maka-Filipino sa ating panahon, na produkto ng isang mas seryoso't mas malalim na sinemang Filipino. Nag-aambag sa seryoso at malalim na sinemang Filipino ang mga politikal na kolektibong pampelikula at maging ang mga susunod na kolektibong naglalayong salungatin ang mga kasinungalingan at panunupil sa lipunan. 


\section{Sanggunian}

Altermidya (People's Alternative Media Network). (2015). About us. Retrieved from http://altermidya.net/ about-us/.

Ang Bayan. (1984, March). Philippines: Communist Party of the Philippines.

Apple Dictionary. (2005-2018). Version 2.3.0. (203.16.12). [Software]. macOS, Apple Inc.

AsiaVisions. (Producer). (1983). Arrogance of power [Video file]. Philippines: AsiaVisions.I

AsiaVisions. (Producer). (1984). Lakbayan '84 [Video file]. Philippines: AsiaVision Media Foundation (AVMF).

AsiaVisions Media Foundation. (1985). Articles of Incorporation. [Microfilm]. Securities and Exchange Commission Public Reference Unit, Mandaluyong City.

AsiaVisions, Christian Aid, \& Tiongson, L. (Producers). (1986). No time for crying [Video file]. Philippines: AsiaVisions Media Foundation (AVMF).

AsiaVisions. (Producer). (1986). Olalia funeral march. [U-matic]. AsiaVisions Audio Visual Collection (Old Code U2 041, U2 042). Ibon Foundation, Quezon City.

AsiaVisions. (Producer). (1987a). Kampo ng bayan. [U-matic S]. AsiaVisions Audio Visual Collection (Old Code U2 028, U2 029, U2 030, U2 031). Ibon Foundation, Quezon City.

AsiaVisions.(Producer).(1987b).Mendiola massacre [Video file].Philippines:AsiaVisions Media Foundation (AVMF).

AsiaVisions. (Producer). (1987c). Partido ng Bayan: Miting de Avance. [U-matic S]. AsiaVisions Audio Visual Collection (Old Code U2 020, U2 021). Ibon Foundation, Quezon City.

AsiaVisions. (Producer). (1987d). Wake at UP Chapel for Lean/Lean's body at hospital/presscon/funeral wake and march/ Funeral march for Lean/Transpo strike at Cubao/CRC puppet presentation. [U-matic S]. AsiaVisions Audio Visual Collection (Old Code U2 057, U2 058, U2 059, U2 060, U2 061, U2 062, U2 063, U2 064, U2 065, U2 066, U2 067). Ibon Foundation, Quezon City.

AsiaVisions, Samahan ng mga Ex-Detainees Laban sa Detensiyon at Aresto, \& Tiongson, L. (Producers). (1987). Beyond the walls of prison [Video file]. Philippines: AsiaVisions.

AsiaVisions. (Producer). (1988). Mendiola Massacre: A year after [Video file]. Philippines: AsiaVisions Media Foundation (AVMF).

AsiaVisions, Cultural Center of the Philippines, \& Tiongson, L. (Producers). (1989). Isang Munting Lupa [Video file]. Philippines: AsiaVisions Media Foundation (AVMF).

AsiaVisions. (Producer). (1991). Fragments [Video file]. Philippines: AsiaVisions Media Foundation (AVMF). AsiaVisions one sheets. (n.d.). [One sheets]. Personal archives of Lito Tiongson. Copy in possession of Mari Luz"Maloy" Quesada-Tiongson.

Bordwell, D. \& Thompson, K. (1997). Film art: An introduction (5th ed.). New York: McGraw-Hill Companies. Community Archiving Workshop Manila Committee. (2017, January). Community Archiving Workshop Manila: A brief. [Digital file]. Unpublished manuscript.

Communication Foundation for Asia. (2018). Milestones in CFA history. Retrieved from https://www. cfamedia.org/cfahistory.html

Coronel, R. (2004, October 19). Sineng bayan sa linggo ng protesta ng mga magbubukid. Retrieved from https://groups.yahoo.com/neo/groups/freefest/conversations/messages/3950. 
Creative Audio-Visual Specialists. (Producer). (1982). Wings of deceit. [Video file] Philippines: Creative Audio-Visual Specialists (CAVS).

Curriculum vitae of Lito Tiongson. (n.d.). [Unpublished manuscript]. Personal archives of Lito Tiongson. Copy in possession of Mari Luz"Maloy" Quesada-Tiongson.

Daroy, P. (1983). Main currents in the Philippine cinema. In N. Tiongson (Ed.), The Urian anthology 19701979 (48-61). Manila: Manuel L. Morato.

Deocampo, N. (1985). Short film: Emergence of a new Philippine cinema. Ed. Alfred A. Yuson. Manila: Communication Foundation for Asia.

Deocampo, N. (1994). Alternative cinema. In N. Tiongson (Ed.), CCP encyclopedia of Philippine art (pp. 5867). Manila: CCP.

Deocampo, N. (2011). Film: American influences on Philippine cinema Mandaluyong City: Anvil Publishing. Edgar Jopson Foundation, \& Alternative Horizons. (Producer). (1986). Edjop [Video file]. Philippines: Alternative Horizons.

Edmondson, R. (2002). Memory of the world: General guidelines to safeguard documentary heritage. Retrieved from http://unesdoc.unesco.org/images/0012 /001256/125637e.pdf.

Edmondson, R. (2004). Audiovisual archiving: Philosophy and principles. Retrieved from http://unesdoc. unesco.org/images /0013/001364/ 136477e.pdf

Fischer, E. (1963). The necessity of art: A Marxist approach. Middlesex, England: Penguin Books.

Gabriel,T.H. (1979). Third Cinema in Third World: The dynamics of style and ideology (Doctoral dissertation). Retrieved from Proquest Dissertations and Theses Database. (Accession Order No. 8001422)

Garcia, H. (1987, November 13-19). Toronto Festival lures $3^{\text {rd }}$ World artists." The Filipino Express, n.p. [Newspaper clipping]. Copy in possession of Mari Luz"Maloy" Quesada-Tiongson.

IBON Foundation. (2013, March 3). New Board of Trustees ushers in IBON's 35h year. Retrieved from https://groups.yahoo.com/neo/groups/ibon/conversations/topics/1148

Iglesias, R. C. (1984, December 16). An emerging People's Cinema. Sunday Malaya.p. 7.

Ilagan, B. (2014, Oktubre 10). Progressive video groups: History, lessons, and challenges [Digitial file titled Kodao Altermidya progressive video groups paper]. Unpublished manuscript. Altermidya: Quezon City

Independent Film and Video Festival. (1986). [Souvenir program]. Copy in possession of Nick Deocampo. Also copy in possession of Cesar Hernando.

Kenny, E. (1996/2005). Making documentaries in the Philippines. Pasig City: Anvil Publishing.

Lacaba, J. (1976, Agosto). Maikling kasaysayan. Sagisag. Retrieved from http://www .manunuri.com/ the_manunuri\#kasaysayan.

Lohr, S. (1984, Mayo 14). Election fairness at issue in Philippine voting. The New York Times. Retrieved from http://www.nytimes.com/1984/05/14/world/elections-fairness-at-issue-in-philippine-votingtoday.html.

Lumbera, B. (2011). Re-viewing Filipino cinema. Mandaluyong City: Anvil Publications.

Mata, E. (1989, February 19). A different film maker. Philippine Daily Globe. n.p. [Clipping of a newspaper]. Copy in possession of Mari Luz"Maloy" Quesada-Tiongson..

Montanez, K. (1988). The new mass art and literature and other related essays: 1974-1987. Quezon City: Kalikasan Press.

Olea, R.V.(2014,October 12). National network of alternative media outfits formed. Bulatlat. Retrieved from 
http://bulatlat.com/main/2014/10/12/national-network-of-alternative-media-outfits-formed/. Phillips, W. H. (2002). Film: An introduction (2nd ed.). Boston: Bedford/St. Martin's.

Roque, R. O. (2016). Sineng bayan: Kasaysayan at filmography ng mga politikal na kolektibong pampelikula (1982-2014) (Unpublished masteral thesis). University of the Philippines, Diliman.

Sison, J. M. (1971/2001). Makibaka para sa pambansang demokrasya. Manila: Amado V. Hernandez Resource Center \& College Editors' Guild of the Philippines.

Solanas, F. \& Gettino, O (1976). Towards a Third Cinema. In B. Nichols (Ed.), Movies and methods (Volume I, pp. 44-64). Berkeley: University of California.

Tiongson, N.G. (ed.). (1984). The politics of culture:The Philippine experience. Manila: Philippine Educational Theatre Association.

Tiongson, N. G. (2001). Introduction: Filipino films in the 1980s. In N. G. Tiongson, The Urian anthology 1980-1989. Manila: Antonio P.Tuviera.

Tolentino, R. B. (2008a). Indie cinema bilang kultural na kapital, KPK Column, Bulatlat. Retrieved from http://rolandotolentino.wordpress .com/2008/08/03/indie-cinema-bilang-kultural-na-kapital-kpkcolumn-bulatlat/.

Tolentino, R. B. (2008b). Mga leksyon mula sa political film collectives sa indie cinema, Cinemalaya 2008 Talk. Retrieved from http://rolandotolentino.wordpress.com/2008/10/09/mga-leksyon-mula-sapolitical-film-collectives-sa-indie-cinema-cinemalaya-2008-talk/.

Tolentino, R. B. (2009a). Political film collectives, Introduction to ASEAC Panel. Retrieved from http:// rolandotolentino.wordpress .com/2009/01/25/political-film-collectives-introduction-to-aseacpanel/.

Tolentino, R. B. (2009b). Akademya at indie filmmaker. Retrieved from http://rolandotolentino.wordpres. com/2009/08/16/akademya-at-indie-filmmaker-kulturang-popular-kultura-column-bulatlat-com/.

Tolentino, R. B. \& Barrios, J. (1994). PAKSA (Panulat para sa Kaunlaran ng Sambayanan). (Literature). In N. G. Tiongson (Ed.), CCP encyclopedia of Philippine art. Vol. IX (p. 694). Manila: CCP.

Torre, N. U. (2013, February 13). From indie to maindie. Philippine Daily Inquirer. Retrieved from http:// entertainment.inquirer.net/30179/from-indie-to-'maindie'.

Villena, G. (2012, July 26). Jose Javier Reyes says yes to "maindie" with indie debut. Philippine News Blog. Retrieved from https://www.yahoo.com/news/blogs/omgphnewsblog/jose-javier-reyes-says-yesmaindie-indie-debut-071109732.html.

Williams, R. (1977). Marxism and literature. Oxford: Oxford University Press.

Young Critics Circle. (2009). Sining ng sineng Filipino. Quezon City: Sentro ng Wikang Filipino-Diliman.

\section{Endnotes}

1 Sa teksbuk na Film: An introduction (Phillips, 2002), ang isang pelikulang independiyente ay sinasabing ginawa nang walang suporta o input mula sa dominante at establisadong industriyang pampelikula.

2 Natukoy man na ang alternatibong pelikula ay kilala ring independiyenteng pelikula, nagbago na sa paglipas ng panahon ang kahulugan ng terminong "independiyente" o "indie" (popular na katawagan sa mga pelikulang independiyente). Sa sanaysay na ito bahagi ang mga nagba(ba)gong pakahulugan sa pelikulang independiyente lalo na sa kasalukuyang panahon sa pagdami ng bilang ng mga pelikulang digital at sa pamamayagpag ng mga film festival na diumano'y nagtatampok 
ng mga gawaing indie. Sa Apple Dictionary Version 2.3.0 (203.16.12), binabanggit na nagsimula ang pagpapaikling ito sa salitang "independent" noong dekada 1920 kaugnay ng produksiyong pampelikula (Apple Dictionary, 2005-2018).

3 Sa karanasan ng mananaliksik-manunulat sa pakikipag-ugnayan sa mga kasapi ng mga kolektibong pampelikulang saklaw ng pag-aaral, lumalabas na hindi kalakaran o hindi nakagawian ng mga kasapi ng mga nasabing KP na tawagin ang kanilang grupo bilang "political film collective." Karaniwang gamit ay"film group" o"video group"tulad halimbawa ng pamagat ng digital file na pinadala ni Bonifacio llagan sa mananaliksik-manunulat na "Kodao Altermidya progressive video groups paper" (B. llagan, personal na komunikasyon, 8 Hunyo 2015). Ito ang pamagat ng digital file ng panayam na "Progressive video groups: History, lessons, and challenges" ni llagan sa kumperensiyang nagbigay-daan sa pagkakatatag ng Altermidya noong 2014.

$4 \quad$ Noong dineklara ang Batas Militar, inaresto si Fr. Cornelio Lagerwey at iba pang nakatataas na opisyal ng Social Communications Center (SSC), ang orihinal na pangalan ng Communication Foundation for Asia (CFA). Ipinasara ang SCC sa ilang panahon at binawi ng lisensiya nito na makapaglimbag (Communication Foundation for Asia, 2018).

5 May ilang artikulo sa pahayagan na nagtala ng taong 1983 (i.e. artikulo sa The Filipino Express ni H. Garcia noong 13-19 Nobyembre 1987) at taong 1985 (i.e. artikulo ni E. Mata sa Philippine Daily Globe noong 19 Pebrero 1989) bilang taon ng pagkakabuo ng AsiaVisions ngunit mas pinapanghawakan ang taong 1982 bilang taon ng pagkakatatag ng AsiaVisions batay sa kauna-unahang pagtatala na nagawa ni Nicanor G. Tiongson na nalathala noong 1984 para sa The politics of culture: The Philippine experience na inilathala ng Philippine Educational Theatre Association (PETA) kaugnay ng proceedings ng MAKIISA 1 People's Culture Festival na isinagawa noong 28-30 Disyembre 1983 sa Dulaang Raha Sulayman, Fort Santiago, Intramuros, Maynila. Ito rin ang taon na itinala ni Nick Deocampo (1994) sa CCP Encyclopedia of Philippine Art kaugnay ng taon ng pagkakatatag ng AsiaVisions.

6 Galing ang datos mula sa mga dokumentong ipinasa ng AsiaVisions sa Securities and Exchange Commission noong 1985. Maaksses ang mga ito, tulad ng Articles of Incorporation (AsiaVisions Media Foundation, 1985) sa Microfilm Section ng Public Reference Unit ng nasabing ahensiya.

7 Halimbawa ang panayam kay Ariel Saturay noong 15 Pebrero 2007 na nagsasaad ng ganitong ugnayan ng AsiaVisions sa AlterHorizons.

8 Sa bahaging ito ng pagsusuma ng moda ng produksiyon, distribusyon, at eksibisyon ng dalawang kolektibong pampelikula, minarapat na ilagay na lamang sa talababa ang mga pinagsaangguniang mga personal na komunikasyon upang hindi makasira sa daloy ng pagbabasa. Laman ng tesis-masteral ng mananaliksik-manunulat na pinamagatang Sineng Bayan: Kasaysayan at filmography ng mga politikal na kolektibong pampelikula (1982-2014) (Roque, 2016) ang detalyadong kasaysayan ng dalawang KP kung saan nabanggit ang lahat ng personal na komunikasyong nagsisilbing sanggunian dito. Sa tesis-masteral na binabanggit hinalaw ang sanaysay na ito.

9 Sa pangkalahatan, nakuha ang mga datos na siyang batayan sa mga paglalahad tungkol sa mga karanasan ng dalawang KP mula sa mga panayam ng mananaliksik-manunulat sa iba't ibang naging kasapi ng AsiaVisions na sina Bonifacio llagan, Danilo Consumido, Rom Dongeto, Jose Clemente, Ariel Saturay, Marlene Francia, Romulo Regalado, Gil Magalong, at Ron Magbuhos Papag at naging kasapi ng AlterHorizons na sina Bobby Roldan, Jose Cuaresma, Monina Bello, Milo Paz, at Cynthia Paz (B. Ilagan, personal na komunikasyon, 12 Marso 2012; D. Consumido, personal na komunikasyon, 22 Marso 2012; 
R. Dongeto, personal na komunikasyon, 20 Marso 2012; J. Clemente, personal na komunikasyon, 8 Mayo 2012; A. Saturay, personal na komunikasyon, Pebrero-Marso 2012; M. Francia, personal na komunikasyon, 9 April 2012; R. Regalado, personal na komunikasyon, 24 Marso 2012; G. Magalong, personal na komunikasyon, 7 April 2012; R. M. Papag, personal na komunikasyon, Marso 2012; B. Roldan, personal na komunikasyon, Marso-Abril 2012; J. Cuaresma, personal na komunikasyon, Marso-Abril 2012; M. Bello, personal na komunikasyon, 16 Hunyo 2012; M. Paz, personal na komunikasyon, 18 Mayo 2012; C. Paz, personal na komunikasyon, 18 Mayo 2012). Dahil namayapa na ang uang executive director na si Lito Tiongson, nagsagawa ng panayam ang mananaliksik-manunulat sa kabiyak ni L.Tiongson na si Mari Luz "Maloy" Quesada-Tiongson (personal na komunikasyon, 11 Marso 2012).

10 Malaking tulong ang entri nina Bordwell at Thompson (1997) sa kanilang librong Film Art: An Introduction (ika-5 ed.) sa pagbuo ng bahaging ito kaugnay ng moda ng kolektibong produksiyon. May tala sina Bordwell at Thompson tungkol sa kolektibong produksiyon batay sa mga karanasan sa kolektibong politikal sa Pranses at Amerika noong dekada 1960 hanggang 1970 na nabanggit na sa paunang bahagi ng sanaysay na ito.

11 Samantala, ang koleksiyon ng AlterHorizons ay nasa personal na pag-iingat ni Jose Cuaresma (personal na komunikasyon, 20 Marso 2012).

12 Mahusay na nagtipon ng proceeding ng kumperensiyang ito ang Arkibong Bayan na orihinal na naglathala sa http://www.arkibongbayan.org/2014/2014-100ct10-altermidya/AlterMidya.htm ng mga panayam at mga litrato kaugnay ng pagtitipon. Gayumpaman, wala sa nasabing online archive ang teksto ng panayam ni Bonifacio llagan. Personal na nabigyan ng kopya ang mananaliksik-manunulat ng panayam bilang digital document file na pinamagatang "Kodao Altermidya progressive video groups paper" (B. Ilagan, personal na komunikasyon, Hunyo 8, 2015). Sa nakapaskil noong 2014 sa Arkibong Bayan, pinamagatan nito ang panayam ni llagan na "Progressive video groups: History, lessons, and challenges." Sa kasamaang-palad din, sa kasalukuyang bersiyon ng online archive, wala na rito ang dokumentasyon nito kaugnay sa First National Conference on Alternative Media. Makikita sa website ng Altermidya ang maikling kasaysayan ng grupo (Altermidya, 2015). Naiulat din sa Bulatlat.com ang kumperensiya (Olea, 2014).

13 Noong isinagawa ang panayam, si Jazminda "Jaz" Lumang ang tumatayong executive director ng IBON. Simula Marso 3, 2013 nagkaroon ng bagong set ng Board of Trustees ang IBON kasama na ang bagong executive director na si Jose Enrique "Sonny" Africa. Si Sonny Africa ang dating research head at miyembro ng Executive Committee ng IBON. Pumalit siya kay Jaz Lumang matapos makapagsilbi nang anim na taon bilang executive director (Ibon Foundation, 2013).

14 Direktang naakses ng mananaliksik-manunulat noong Abril hanggang Mayo 2012 ang mga nasabing U-matic tape at iba pang nakasaad dito dahil sa pagpapahintulot ng lbon Foundation noong nasa pamumuno ito ni Jaz Lumang, nakalipas na executive director ng Ibon, na makapagsagawa ng pananaliksik sa pamamagitan ng pag-iimbentaryo ng mga koleksiyong bahagi ng arkibong awdyobiswal ng AsiaVisions.

15 Sa pagsagawa ng mananaliksik-manunulat ng imbentaryo ng AsiaVisions AV Collection noong mga buwan ng Abril hanggang Mayo 2012 at sa proseso ng tuloy-tuloy na Community Archiving Workshop project sa koleksiyon, makikita sa database ang mga kopya ng ilan sa gawa ng AlterHorizons na nasa koleksiyon ng AsiaVisions.

16 Ang PAKSA o Panulat para sa Kaunlaran ng Sambayanan ay itinatag noong 23 Marso 1971 
sa Polytechnic College of Commerce (Polytechnic University of the Philippines na sa kasalukuyan). Tugon ito sa rebolusyonaryong kalagayan $\mathrm{ng}$ mga panahon iyon at nagsulong $\mathrm{ng}$ demokratikong rebolusyonaryong kultura. Saglit lamang nabuhay ang organisasyon. Gayumpaman, ang konsepto nito ng isang pambansa demokratikong panitikan ay hindi namatay at patuloy na lumaganap sa hanay ng mga baryo, maralitang komunidad, paaralan, at pabrika kung saan patuloy ang paglaban sa pang-aapi (Tolentino \& Barrios, 1994).

17 Tumutukoy ang Manununuri sa Manunuri ng Pelikulang Pilipino o MPP. Ayon sa opisyal na website nito kaugnay ng maikling kasaysayan ng pagkakatatag ng grupo (Lacaba, 1976, para. 1):"Sa Taza de Oro sa Roxas Boulevard, noong Mayo 1, 1976, nagtipon-tipon ang siyam na katao at pinag-usapan ang pagbibigay ng taunang gawad sa mga pelikulang Filipino. Ang siyam ay sina Pio de Castro, Behn Cervantes, Pete Daroy, Mario Hernando, Bien Lumbera, Doy del Mundo, Manny Pichel, Nic Tiongson, at Nestor Torre. Hindi nakarating sa pulong si Gino Dormiendo, ngunit siya'y nagpahayag ng pagsang-ayon sa mga layunin ng grupo nang siya'y kausapin pagkaraan ng unang pulong."

ROSEMARIE O. ROQUE obtained her degree in MA Araling Pilipino from UP Diliman in 2016 and is an Assistant Professor of the Department of Arts and Communication (DAC) in UP Manila. She is an associate member of the South East Asia Pacific Audio Visual Archives Association (SEAPAVAA). Representing the Society of Filipino Archivists for Film (SOFIA), she is currently the assistant secretary of the National Committee on Archives (NCA) of the National Commission for Culture and the Arts (NCCA). (corresponding author: roroque@up.edu.ph) 